\title{
Hidden gauge structure of supersymmetric free differential algebras
}

\author{
Laura Andrianopoli, ${ }^{a, b}$ Riccardo D'Auria $^{a}$ and Lucrezia Ravera ${ }^{a, b}$ \\ ${ }^{a}$ DISAT, Politecnico di Torino, \\ Corso Duca degli Abruzzi 24, I-10129 Turin \\ ${ }^{b}$ INFN - Sezione di Torino, \\ Torino, Italy \\ E-mail: laura.andrianopoli@polito.it, riccardo.dauria@polito.it, \\ lucrezia.ravera@polito.it
}

ABSTRACT: The aim of this paper is to clarify the role of the nilpotent fermionic generator $Q^{\prime}$ introduced in [6] and appearing in the hidden supergroup underlying the free differential algebra (FDA) of $\mathrm{D}=11$ supergravity.

We give a physical explanation of its role by looking at the gauge properties of the theory. We find that its presence is necessary, in order that the extra 1-forms of the hidden supergroup give rise to the correct gauge transformations of the $p$-forms of the FDA. This interpretation is actually valid for any supergravity containing antisymmetric tensor fields, and any supersymmetric FDA can always be traded for a hidden Lie superalgebra containing extra fermionic nilpotent generators.

As an interesting example we construct the hidden superalgebra associated with the FDA of $\mathcal{N}=2, D=7$ supergravity. In this case we are able to parametrize the mutually non local 2- and 3-form $B^{(2)}$ and $B^{(3)}$ in terms of hidden 1-forms and find that supersymmetry and gauge invariance require in general the presence of two nilpotent fermionic generators in the hidden algebra.

We propose that our approach, where all the invariances of the FDA are expressed as Lie derivatives of the $p$-forms in the hidden supergroup manifold, could be an appropriate framework to discuss theories defined in enlarged versions of superspace recently considered in the literature, such us double field theory and its generalizations.

KEYwords: Supergravity Models, Gauge Symmetry, p-branes, Supersymmetry and Duality

ARXIV EPRINT: 1606.07328 


\section{Contents}

1 Introduction 1

2 Review of the eleven dimensional hidden superalgebra 5

3 FDA gauge structure and supergravity $\quad 9$

3.1 Gauge transformations from the hidden supergroup manifold 11

$\begin{array}{ll}3.2 & \text { The role of the nilpotent fermionic generator } Q^{\prime} \\ \end{array}$

4 The hidden gauge algebra of $D=7, \mathcal{N}=2$ Free Differential Algebra 15

$\begin{array}{lll}4.1 & \text { The hidden superalgebra } & 19\end{array}$

4.2 Including $B_{a_{1} \cdots a_{5}} \quad 21$

4.3 Gauge structure of the minimal $D=7 \mathrm{FDA} \quad 22$

5 Relation with eleven dimensional supergravity 24

6 Concluding remarks $\quad 26$

A The explicit solution for $A^{(3)}$ in $D=11 \quad 28$

B The explicit solution for $B^{(2)}$ and $B^{(3)}$ in $D=7 \quad 28$

B.1 Coefficients in the case $B_{a_{1} \cdots a_{5}}=0 \quad 28$

B.2 Coefficients in the case $B_{a_{1} \cdots a_{5}} \neq 0 \quad 29$

C Dimensional reduction of the gamma matrices $\quad 30$

C.1 Properties of the 't Hooft matrices 31

D Fierz identities and irreducible representations $\quad 31$

D.1 3-gravitino irreducible representations in $D=11$

D.2 Irreducible representations in $D=7 \quad 32$

\section{Introduction}

Supergravity theories in various space-time dimensions $4 \leq D \leq 11$ have a bosonic field content that generically includes, besides the metric and a set of 1-form gauge potentials, also $(p+1)$-form gauge potentials of various $p \leq 9$, and they are therefore appropriately discussed in the context of Free Differential Algebras (FDA in the following). This is also required from superstring theories, where the higher form potentials are related to the NS-NS and R-R sectors of the different superstring theories (as general references on superstring theory, see [1-4]). 
Early after the discovery of Supergravity, the action of $D=11$ supergravity was first constructed in [5]. It has a bosonic field content given by the metric $g_{\mu \nu}$ and a 3 -index antisymmetric tensor $A_{\mu \nu \rho}(\mu, \nu, \rho, \cdots=0,1, \cdots, D-1)$, together with a single Majorana gravitino $\Psi_{\mu}$ in the fermionic sector.

The structure of this same theory was reconsidered in [6] in the framework of FDAs using the superspace geometric approach. In this setting, its bosonic sector includes, besides the supervielbein $\left\{V^{a}, \Psi\right\}$, a 3 -form potential $A^{(3)}$, with field-strength $F^{(4)}=$ $d A^{(3)}$ (modulo gravitino 1-form bilinears), together with its Hodge-dual $F^{(7)}$, defined such that its space-time components are related to the ones of the 4 -form by $F_{\mu_{1} \cdots \mu_{7}}=$ $\frac{1}{84} \epsilon_{\mu_{1} \cdots \mu_{7} \nu_{1} \cdots \nu_{4}} F^{\nu_{1} \cdots \nu_{4}}$; this amounts to say that it is associated with a 6 -form potential $B^{(6)}$ in superspace. The on-shell closure of the supersymmetric theory relies on 3-fermions Fierz identities and requires $F^{(7)}=d B^{(6)}-15 A^{(3)} \wedge F^{(4)}$ (modulo fermionic currents).

In the same paper the supersymmetric FDA was also investigated in order to see whether the FDA formulation could be interpreted in terms of an ordinary Lie superalgebra in its dual Maurer-Cartan formulation. Actually, this was proven to be true and the existence of a superalgebra underlying the theory was presented for the first time.

This superalgebra includes as a subalgebra the super-Poincaré algebra of the eleven dimensional theory, but it also contains two extra bosonic generators $Z^{a b}, Z^{a_{1} \cdots a_{5}}(a, b, \cdots=$ $0,1, \cdots 10$ ), which commute with the 4-momentum $P_{a}$, while having appropriate commutators with the eleven dimensional Lorentz generators $J_{a b}{ }^{1}$ In the following, generators that commute with all the superalgebra but the Lorentz generators will be named "almost central". Furthermore, to close the algebra, an extra nilpotent fermionic generator called $Q^{\prime}$ must be included.

Besides the standard Poincaré Lie algebra, the superalgebra associated with $D=11$ supergravity has the following structure of (anti)commutators:

$$
\begin{aligned}
\{Q, Q\} & =-\mathrm{i} C \Gamma^{a} P_{a}-\frac{1}{2} C \Gamma_{a b} Z^{a b}-\frac{\mathrm{i}}{5 !} C \Gamma_{a_{1} \cdots a_{5}} Z^{a_{1} \cdots a_{5}} ; \\
{\left[Q, P_{a}\right] } & \propto \Gamma_{a} Q^{\prime} ; \\
{\left[Q, Z^{a b}\right] } & \propto \Gamma^{a b} Q^{\prime} \\
{\left[Q, Z^{a_{1} \cdots a_{5}}\right] } & \propto \Gamma^{a_{1} \cdots a_{5}} Q^{\prime} ; \\
\left\{Q^{\prime}, Q^{\prime}\right\} & =0
\end{aligned}
$$

together with

$$
\begin{aligned}
{\left[J_{a b}, Z^{c d}\right] } & \propto \delta_{[a}^{[c} \eta_{b] l} Z^{d] l} ; & {\left[J_{a b}, Z^{c_{1} \cdots c_{5}}\right] } & \propto \delta_{[a}^{\left[c_{1}\right.} \eta_{b] l} Z^{\left.c_{2} \cdots c_{5}\right] l} \\
{\left[J_{a b}, Q\right] } & \propto \Gamma_{a b} Q ; & {\left[J_{a b}, Q^{\prime}\right] } & \propto \Gamma_{a b} Q^{\prime},
\end{aligned}
$$

the other (anti)commutation relations being zero. The precise relations are reported in section 2. Here and in the following we shall refer to a superalgebra descending from a given FDA as a hidden superalgebra. Note that the set of generators $\left\{Z^{a b}, Z^{a_{1} \cdots a_{5}}, Q^{\prime}\right\}$, extending

\footnotetext{
${ }^{1}$ They are indeed 1 -forms valued in the antisymmetric tensor represemtations of $\operatorname{SO}(1,10)$.
} 
the super-Poincaré Lie algebra to the hidden superalgebra written above, actually span an abelian ideal of it. They will also be referred to as hidden generators.

Let us remark that the anticommutation relation (1.1) generalizes to almost central charges the central extension of the supersymmetry algebra [7], which was shown in [8] to be associated with topologically non trivial configurations of the bosonic fields. The possible extension (1.1) of the supersymmetry algebra, for supergravity theories in $D>4$ dimensions, was later widely considered (see in particular [9]-[12]). After the discovery of $\mathrm{D} p$-branes as sources for the R-R gauge potentials [13] and the ensuing understanding of the duality relation between eleven dimensional supergravity and Type IIA theory in ten dimensions, the bosonic generators $Z^{a b}, Z^{a_{1} \cdots a_{5}}$ were understood as $p$-brane charges, sources of the dual potentials $A_{(3)}$ and $B_{(6)}[14,15]$, and eq. (1.1) was then interpreted as the natural generalization of the supersymmetry algebra in higher dimensions, in the presence of non-trivial topological extended sources (black $p$-branes).

However, the structure of the full superalgebra, given in eq.s (1.1)-(1.5), which is hidden in the superymmetric $\mathrm{D}=11 \mathrm{FDA}$, besides the almost central charges $Z^{a b}$ and $Z^{a_{1} \cdots a_{5}}$, also requires for its consistency (closure of the super-Jacobi identities) the presence of an extra fermionic nilpotent charge, $Q^{\prime}$, as shown in reference [6]. This fact is not a peculiarity of the eleven dimensional theory, but is fully general, and, as we will extensively discuss in this paper, a hidden superalgebra underlying the supersymmetric FDA containing at least one nilpotent fermionic generator can be constructed for each supergravity theory where antisymmetric tensor fields are present.

The role played by the extra fermionic generator $Q^{\prime}$ and its group-theoretical and physical meaning, corresponding to the non-trivial contributions (1.2)-(1.5), was much less investigated with respect to that of the almost central charges. The most relevant contributions that we are aware of were given first in [9] and then in particular in $[16,17]$, where the results in [6] were further analyzed and generalized. However, the physical meaning of $Q^{\prime}$ remained obscure, at our knowledge.

Actually, the consistency of the $D=11$ theory, that is the closure of the supersymmetric FDA and of its hidden superalgebra, fully relies on 3-fermion Fierz identities obeyed by the gravitino 1-forms, and it crucially requires the presence of the nilpotent spin-3/2 field $\eta$ associated with the fermionic charge $Q^{\prime}$. Three-gravitini Fierz-identities are at the heart of the closure of all lower dimensional supergravities, and in particular of those based on FDA's. As a consequence of this, almost central-extended hidden superalgebras, including extra nilpotent fermionic generators as necessary ingredients, should underly all the supergravity theories based on FDAs, as we have explicitly checked in various supergravity models with $6 \leq D \leq 9$.

It is the aim of the present paper to further investigate the superalgebra hidden in all the supersymmetric FDAs and to clarify the role played by the bosonic and fermionic generators. In particular, we will analyze in detail the gauge structure of the supersymmetric FDA in eleven dimensions in relation to its hidden gauge superalgebra, and then we will consider a specific case in lower dimensions (we will choose minimal supergravity in $\mathrm{D}=7$ ) to test the universality of the construction and to investigate possible extensions of the underlying superalgebra of [6]. 
The main result of our paper is to disclose the physical interpretation of the fermionic hidden generator $Q^{\prime}$. We will show that it has a topological meaning, since it controls the gauge structure of the FDA once it is expressed in terms of 1-forms. We will also find that in general more than one nilpotent fermionic generator are necessary to construct the fully extended superalgebra hidden in the supersymmetric FDA. This will be the case in particular of the minimal supersymmetric $D=7 \mathrm{FDA}$, which we will analyze in some detail.

Considering now the bosonic hidden generators of the hidden algebra (we will call $H_{b}$ the corresponding tangent space directions of the hidden group manifold), we will show that they are associated with internal diffeomorphisms of the supersymmetric FDA in $D$ dimensions. More precisely, once a $p$-form $A^{(p)}$ of the FDA is parametrized in terms of the hidden 1-forms, contraction of $A^{(p)}$ along a generic tangent vector $\vec{z} \in H_{b}$ gives a $(p-1)$ form gauge parameter, and the Lie derivative of the FDA along a tangent vector $\vec{z}$ gives a gauge transformation leaving the FDA invariant.

This construction is not limited to the eleven dimensional FDA. In particular, it is interesting to consider ten dimensional Type IIA supergravity, which naturally descends from the $D=11$ theory. Its FDA includes the 2-form NS-NS field $B^{(2)}$, also appearing in all superstring-related supergravities, which has a natural understanding in terms of the antisymmetric 3-form $A^{(3)}$ of $\mathrm{D}=11$ supergravity. The corresponding hidden 1-form field, $B_{a}$, has an associated charge $Z^{a}$ which carries a Lorentz-index, contravariant with respect to the one carried by the translation generator $P_{a}$. It follows that in the fully extended hidden superalgebra in any $D \leq 10, P_{a}$ and $Z^{a}$ appear on the same footing and the action of the hidden superalgebra in this case includes automorphisms interchanging them. When some of the space-time directions are compactified on circles, these automorphisms are naturally associated with T-duality transformations interchanging momentum with winding in the compact directions.

As we are going to discuss in the following, the structure outlined above is strongly reminiscent of the one described in the framework of generalized geometry [18]-[23] and its extensions to M-theory [24]-[26], double field theory [27]-[32] and exceptional field theory [33]-[35]. We expect that our formalism could be useful in this context.

To clarify the crucial role played by the nilpotent hidden fermionic generators for the consistency of the hidden superalgebra, we will consider a singular limit where the associated spinor 1-form $\eta$ satisfies $\eta \rightarrow 0$. In this limit the supersymmetric FDA parametrized in terms of 1-forms becomes ill defined: indeed the exterior forms $A^{(p)}$ are gauge fields, since they include "longitudinal" unphysical directions corresponding to the gauge freedom $A^{(p)} \rightarrow A^{(p)}+d \Lambda^{(p-1)}$. In the limit $\eta \rightarrow 0$, the unphysical degrees of freedom $\Lambda^{(p-1)}$ get mixed with the physical directions of the superspace, and all the generators of the hidden superalgebra act as generators of external diffeomorphisms. On the contrary, when $\eta \neq 0$ the hidden supergroup acquires a principal fiber bundle structure; $\eta$ allows to separate, in a dynamical way, the physical directions of superspace, generated by the super-vielbein $\left(V^{a}, \Psi\right)$, from the other directions, belonging to the fiber of superspace, in such a way as to recover the gauge invariance of the FDA.

In this paper we will limit ourselves to consider the FDA and its underlying supergroup, corresponding to the ground state of the supergravity theory, also referred to as the 
"vacuum", which is defined by the condition that all the supercurvatures vanish, so that only the topological structure and the symmetries of the theory emerge. As is usual in supersymmetric theories, they include, besides the local symmetries which can be realized at the lagrangian level, also non-perturbative symmetries, associated with mutually nonlocal generators. We will not consider here the full dynamical content of the theory out of the vacuum, where the simultaneous presence of mutually non-local electric and magnetic $p$-forms is forbidden at the lagrangian level. ${ }^{2}$ For the $\mathrm{D}=7$ theory under consideration, we will show that it is however possible to find two inequivalent "Lagrangian subalgebras" of the hidden superalgabra, which only include mutually local fields and which should be relevant for the Lagrangian description of the interacting theory. Actually, each of them includes, as hidden fermionic generators, only one of the two nilpotent spinors.

The paper is organized as follows.

In section 2 we will review, in a critical way, the various steps of the construction of the superalgebra hidden in eleven dimensional supergravity, following [6].

Then, in section 3 we will analyze in detail the gauge structure of the hidden superalgebra, discussing in particular the role of the nilpotent generator in the $\mathrm{D}=11$ supersymmetric FDA.

In section 4 we will focus our study on the minimal $D=7, \mathcal{N}=2$ supergravity theory, whose FDA is particularly rich since it includes, besides a triplet of gauge vectors $A^{x}$, a 2 -form $B^{(2)}$, a 3 -form $B^{(3)}$ related to $B^{(2)}$ by Hodge-duality of the corresponding field strengths, and a triplet of 4 -forms $A^{x \mid(4)}$ related to $A^{x}$ by Hodge-duality of the corresponding field strengths. This theory can be obtained by dimensional reduction, on a four-dimensional compact manifold preserving only half of the supersymmetries, from $D=11$ supergravity. We will provide the parametrization in terms of 1 -forms of the mutually non local fields $B^{(2)}$ and $B^{(3)}$, finding the corresponding superalgebra hidden in the supersymmetric FDA. Actually in this case we will find that two nilpotent fermionic generators are required for the closure of the fully extended hidden superalgebra.

In section 5 we will consider the conditions under which the seven dimensional model studied in section 4 could be obtained by dimensional reduction of the eleven dimensional model of section 2 .

The main body of the paper ends in section 6 with some concluding remarks. Our notations and conventions, together with some technical details, can be found in the appendices.

\section{Review of the eleven dimensional hidden superalgebra}

As said in the introduction, the $D=11$ theory, first constructed in [5], was reformulated in ref. [6] using a geometric superspace approach, in terms of a supersymmetric FDA. ${ }^{3}$ In this context the bosonic vielbein $V^{a}(a=0,1, \cdots, 10)$, together with the gravitino 1-form $\Psi$,

\footnotetext{
${ }^{2}$ Some progress in this topic has been obtained in reference $[16,17]$.

${ }^{3}$ In the original paper [6] the FDA was referred to as Cartan Integrable System (CIS), since the authors were unaware of the previous work by Sullivan [36] who actually introduced the mathematical concept of FDA to which the CIS are equivalent.
} 
span a basis of the cotangent superspace $K \equiv\left\{V^{a}, \Psi\right\}$, where also the superspace 3 -form $A^{(3)}$, whose pull-back on space-time is $A_{\mu \nu \rho}$, is defined.

Actually, it was stressed there that besides the simplest FDA including as exterior form only $A^{(3)}$, one can fully extend the FDA to include also a (magnetic) 6-form potential $B^{(6)}$, related to $A^{(3)}$ by Hodge-duality of the corresponding field-strengths. More precisely, the supersymmetric FDA, which defines the ground state of the theory, is given by the vanishing of the following set of supercurvatures:

$$
\begin{aligned}
R^{a b} & \equiv d \omega^{a b}-\frac{1}{2} \omega^{a c} \wedge \omega^{b d} \eta_{c d}=0, \\
T^{a} & \equiv D V^{a}-\frac{\mathrm{i}}{2} \bar{\Psi} \wedge \Gamma^{a} \Psi=0, \\
\rho & \equiv D \Psi=0, \\
F^{(4)} & \equiv d A^{(3)}-\frac{1}{2} \bar{\Psi} \wedge \Gamma_{a b} \Psi \wedge V^{a} \wedge V^{b}=0, \\
F^{(7)} & \equiv d B^{(6)}-15 A^{(3)} \wedge d A^{(3)}-\frac{\mathrm{i}}{2} \bar{\Psi} \wedge \Gamma_{a_{1} \cdots a_{5}} \Psi \wedge V^{a_{1}} \wedge \cdots V^{a_{5}}=0,
\end{aligned}
$$

where $D$ denotes the eleven dimensional Lorentz-covariant derivative and its closure $d^{2}=0$ is a consequence of 3 -fermions Fierz identities in eleven dimensions (see appendix D). ${ }^{4}$ The interacting theory (out of the ground state), including the field equations, is obtained in this setting through a straightforward procedure [6, 37], corresponding to introducing a non-vanishing value to the super-curvatures defined in the left-hand side of the FDA, and given respectively by the super Riemann 2 -form $R^{a b}$, the supertorsion $T^{a}$, the gravitino super field-strength $\rho$, the 4 -form $F^{(4)}$ and its Hodge-dual on space-time $F^{(7)}$. We will not further elaborate on this, here, since the topological structure of the theory, which will be the object of the present investigation, is fully catched by the ground state FDA.

The authors of [6] asked themselves whether one could trade the FDA structure on which the theory is based with an ordinary Lie superalgebra, written in its dual Cartan form, that is in terms of 1-form gauge fields which turn out to be valued in non trivial tensor representations of Lorentz group $\mathrm{SO}(1,10)$. This would allow to disclose the fully extended superalgebra hidden in the supersymmetric FDA.

It was found that this is indeed possible by associating, to the forms $A^{(3)}$ and $B^{(6)}$, the bosonic 1-forms $B_{a b}$ and $B_{a_{1} \cdots a_{5}}$, in the antisymmetric representations of $\mathrm{SO}(1,10)$, whose Maurer-Cartan equations are:

$$
\begin{aligned}
D B_{a_{1} a_{2}} & =\frac{1}{2} \bar{\Psi} \wedge \Gamma_{a_{1} a_{2}} \Psi \\
D B_{a_{1} \ldots a_{5}} & =\frac{\mathrm{i}}{2} \bar{\Psi} \wedge \Gamma_{a_{1} \ldots a 5} \Psi
\end{aligned}
$$

$D$ being the Lorentz-covariant derivatives. In particular, they presented a general decomposition of the 3 -form $A^{(3)}$ in terms of the 1-forms $B_{a b}$ and $B_{a_{1} \ldots a_{5}}$, by requiring the Bianchi identities in superspace of the 3 -form, $d^{2} A^{(3)}=0$, to be satisfied also when $A^{(3)}$

\footnotetext{
${ }^{4}$ In the ground state the spin- $1 / 2$ fields are zero by Lorentz invariance and the scalar fields are constant (they can be set to zero).
} 
is decomposed in terms of the 1 -forms $B_{a b}$ and $B_{a_{1} \ldots a_{5}}$. Actually, it was shown that this program can be accomplished if and only if, together with the newly introduced bosonic 1-form fields, one also introduces an extra spinor 1-form $\eta$, satisfying:

$$
D \eta=\mathrm{i} E_{1} \Gamma_{a} \Psi \wedge V^{a}+E_{2} \Gamma^{a b} \Psi \wedge B_{a b}+\mathrm{i} E_{3} \Gamma^{a_{1} \ldots a_{5}} \Psi \wedge B_{a_{1} \ldots a_{5}} .
$$

They found that the most general solution enjoying the above requirements has the following form: ${ }^{5}$

$$
\begin{aligned}
A^{(3)}= & T_{0} B_{a b} \wedge V^{a} \wedge V^{b}+T_{1} B_{a b} \wedge B_{c}^{b} \wedge B^{c a} \\
& +T_{2} B_{b_{1} a_{1} \ldots a_{4}} \wedge B_{b_{2}}^{b_{1}} \wedge B^{b_{2} a_{1} \ldots a_{4}}+T_{3} \epsilon_{a_{1} \ldots a_{5} b_{1} \ldots b_{5} m} B^{a_{1} \ldots a_{5}} \wedge B^{b_{1} \ldots b_{5}} \wedge V^{m} \\
& +T_{4} \epsilon_{m_{1} \ldots m_{6} n_{1} \ldots n_{5}} B^{m_{1} m_{2} m_{3} p_{1} p_{2}} \wedge B^{m_{4} m_{5} m_{6} p_{1} p_{2}} \wedge B^{n_{1} \ldots n_{5}} \\
& +\mathrm{i} S_{1} \bar{\Psi} \wedge \Gamma_{a} \eta \wedge V^{a}+S_{2} \bar{\Psi} \wedge \Gamma^{a b} \eta \wedge B_{a b}+\mathrm{i} S_{3} \bar{\Psi} \wedge \Gamma^{a_{1} \ldots a_{5}} \eta \wedge B_{a_{1} \ldots a_{5}}
\end{aligned}
$$

where the requirement that $A^{(3)}$ in (2.9) satisfies eq. (2.4) fixes the free constants $T_{i}, S_{j}$ in terms of the structure constants $E_{1}, E_{2}, E_{3}$. Actually, the consistence of the theory also requires the $d^{2}$ closure of the newly introduced fields $B_{a b}, B_{a_{1} \cdots a_{5}}$ and $\eta$. For the two bosonic 1-form fields the $d^{2}$ closure is obvious in the ground state, because of the vanishing of the curvatures $R^{a b}$ and $\rho$, while on $\eta$ it requires the further condition:

$$
E_{1}+10 E_{2}-720 E_{3}=0
$$

The final result is:

$$
\begin{array}{rlrl}
T_{0} & =\frac{120 E_{3}^{2}}{\left(E_{2}-60 E_{3}\right)^{2}}+\frac{1}{6}, & T_{1}=-\frac{E_{2}\left(E_{2}-120 E_{3}\right)}{90\left(E_{2}-60 E_{3}\right)^{2}}, & T_{2}=-\frac{5 E_{3}^{2}}{\left(E_{2}-60 E_{3}\right)^{2}}, \\
T_{3}=\frac{E_{3}^{2}}{120\left(E_{2}-60 E_{3}\right)^{2}}, & T_{4}=-\frac{E_{3}^{2}}{216\left(E_{2}-60 E_{3}\right)^{2}}, & S_{1}=\frac{E_{2}-48 E_{3}}{24\left(E_{2}-60 E_{3}\right)^{2}}, \\
S_{2}=-\frac{E_{2}-120 E_{3}}{240\left(E_{2}-60 E_{3}\right)^{2}}, & S_{3}=\frac{E_{3}}{240\left(E_{2}-60 E_{3}\right)^{2}}, & \\
E_{1}=-10\left(E_{2}-72 E_{3}\right) . & & &
\end{array}
$$

where the constants $E_{1}, E_{2}, E_{3}$ define new structure constants of the hidden super-algebra.

In [6] the first coefficient $T_{0}$ was arbitrarily fixed to $T_{0}=1$ giving only 2 possible solutions for the set of parameters $\left\{T_{i}, S_{j}, E_{k}\right\}$. It was pointed out later in $[16,17]$ that this restriction can be relaxed thus giving the general solution (2.11). Indeed, as observed in the quoted reference, one of the $E_{i}$ can be reabsorbed in the normalization of $\eta$, so that, owing to the relation (2.8), we are left with one free parameter, say $E_{3} / E_{2} .{ }^{6}$ The details of the calculation are reported in appendix A, where also some misprints of [6], in part recognized already in $[16,17]$, are corrected.

\footnotetext{
${ }^{5}$ Here, and in the following, with $B_{a_{1} \ldots a_{p-1}}{ }^{b}$ we generally mean $B_{a_{1} \ldots a_{p}} \eta^{b a_{p}}$, where $\eta_{a b}=(+,-, \cdots,-)$ denotes the Minkowski metric.

${ }^{6}$ In reference $[16,17]$ their free parameter $s$ is different from ours and is related to $E_{3} / E_{2}=\rho$ by the relation $\frac{120 \rho-1}{90(60 \rho-1)^{2}}=\frac{2(3+s)}{15 s^{2}}$.
} 
The full Maurer-Cartan equations of the hidden algebra (in dual form) are then:

$$
\begin{aligned}
d \omega^{a b} & =\frac{1}{2} \omega^{a c} \wedge \omega^{b d} \eta_{c d} \\
D V^{a} & =\frac{\mathrm{i}}{2} \bar{\Psi} \wedge \Gamma^{a} \Psi \\
D \Psi & =0 \\
D B_{a_{1} a_{2}} & =\frac{1}{2} \bar{\Psi} \wedge \Gamma_{a_{1} a_{2}} \Psi, \\
D B_{a_{1} \ldots a_{5}} & =\frac{\mathrm{i}}{2} \bar{\Psi} \wedge \Gamma_{a_{1} \ldots a_{5}} \Psi, \\
D \eta & =\mathrm{i} E_{1} \Gamma_{a} \Psi \wedge V^{a}+E_{2} \Gamma^{a b} \Psi \wedge B_{a b}+\mathrm{i} E_{3} \Gamma^{a_{1} \ldots a_{5}} \Psi \wedge B_{a_{1} \ldots a_{5}} .
\end{aligned}
$$

Let us finally write down the hidden superalgebra in terms of generators closing a set of (anti)commutation relations. For a generic set of 1-forms $\sigma^{\Lambda}$ satisfying the Maurer-Cartan equations:

$$
d \sigma^{\Lambda}=-\frac{1}{2} C_{\Sigma \Gamma}^{\Lambda} \sigma^{\Sigma} \wedge \sigma^{\Gamma}
$$

in terms of structure constants $C_{\Sigma \Gamma}^{\Lambda}$, this is performed by introducing a set of dual generators $T_{\Lambda}$ satisfying

$$
\sigma^{\Lambda}\left(T_{\Sigma}\right)=\delta_{\Sigma}^{\Lambda} ; \quad d \sigma^{\Lambda}\left(T_{\Sigma}, T_{\Gamma}\right)=C_{\Sigma \Gamma}^{\Lambda}
$$

so that the $\left\{T_{\Lambda}\right\}$ close the algebra $\left[T_{\Sigma}, T_{\Gamma}\right]=C^{\Lambda}{ }_{\Sigma \Gamma} T_{\Lambda}$.

In the case at hand, the 1 -forms $\sigma^{\Lambda}$ are

$$
\sigma^{\Lambda} \equiv\left\{V^{a}, \Psi, \omega^{a b}, B_{a b}, B_{a_{1} \ldots a_{5}}, \eta\right\}
$$

To recover the superalgebra in terms of (anti)-commutators of the dual Lie superalgebra generators:

$$
T_{\Lambda} \equiv\left\{P_{a}, Q, J_{a b}, Z^{a b}, Z^{a_{1} \ldots a_{5}}, Q^{\prime}\right\}
$$

we use the duality between 1 -forms and generators defined by the usual conditions:

$$
\begin{aligned}
V^{a}\left(P_{b}\right) & =\delta_{b}^{a}, & \Psi(Q) & =\mathbb{1}, & \omega^{a b}\left(J_{c d}\right) & =2 \delta_{c d}^{a b}, \\
B^{a b}\left(Z_{c d}\right) & =2 \delta_{c d}^{a b}, & B^{a_{1} \ldots a_{5}}\left(Z_{b_{1} \ldots b_{5}}\right) & =5 ! \delta_{b_{1} \ldots b_{5}}^{a_{1} \ldots a_{5}}, & \eta\left(Q^{\prime}\right) & =\mathbb{1}
\end{aligned}
$$

where $\mathbb{1}$ denotes unity in the spinor representation. The $D=11 \mathrm{FDA}$ then corresponds to the following hidden contributions to the superalgebra (besides the Poincaré algebra):

$$
\begin{aligned}
\{Q, \bar{Q}\} & =-\left(\mathrm{i} \Gamma^{a} P_{a}+\frac{1}{2} \Gamma^{a b} Z_{a b}+\frac{\mathrm{i}}{5 !} \Gamma^{a_{1} \ldots a_{5}} Z_{a_{1} \ldots a_{5}}\right) \\
\left\{Q^{\prime}, \bar{Q}^{\prime}\right\} & =0 \\
{\left[Q, P_{a}\right] } & =-2 \mathrm{i} E_{1} \Gamma_{a} Q^{\prime} \\
{\left[Q, Z^{a b}\right] } & =-4 E_{2} \Gamma^{a b} Q^{\prime}
\end{aligned}
$$




$$
\begin{aligned}
{\left[Q, Z^{a_{1} \ldots a_{5}}\right] } & =-2(5 !) \mathrm{i} E_{3} \Gamma^{a_{1} \ldots a_{5}} Q^{\prime} \\
{\left[J_{a b}, Z^{c d}\right] } & =-8 \delta_{[a}^{[c} Z_{b]}^{d]} \\
{\left[J_{a b}, Z^{c_{1} \ldots c_{5}}\right] } & =-20 \delta_{[a}^{\left[c_{1}\right.} Z_{b]}^{\left.c_{2} \ldots c_{5}\right]}, \\
{\left[J_{a b}, Q\right] } & =-\Gamma_{a b} Q \\
{\left[J_{a b}, Q^{\prime}\right] } & =-\Gamma_{a b} Q^{\prime} .
\end{aligned}
$$

All the other commutators (beyond the Poincaré part) vanishing. As said before, the $E_{i}$ satisfy equation (2.10) and one of them can be reabsorbed in the normalization of the $\eta 1$-form. ${ }^{7}$

Finally, let us recall that the presence of the bosonic hidden 1-forms $B_{a b}, B_{a_{1} \ldots a_{5}}$ in the relation (2.22), which generalizes the centrally extended supersymmetry algebra of [8] (where the central generators were associated with electric and magnetic charges), has in fact a topological meaning. This was recognized in [10] and [11], where it was shown they to be associated with extended objects (2-brane and 5-brane) in space-time. In particular in reference [11] it was shown that quite generally such p-forms must be present in any dimensions, their associated (almost) central charges appearing in the supersymmetry algebra. As we shall see, this in fact occurs in the minimal $D=7$ theory that we shall analyze in section 4. The results of [6], and those of [10] and [11] can thus be considered an important extension of the property found in [8].

On the other hand, the fact that the supersymmetry algebra, once extended to its hidden superalgebra, requires the presence of extra spinor generators, was not discussed in $[10,11]$. As we are going to discuss in the next section, the presence of nilpotent fermionic charges in the hidden sector has instead a crucial role for the consistency of the FDA in superspace.

\section{$3 \quad$ FDA gauge structure and supergravity}

The aim of this section is to analyze in detail the hidden gauge structure of the FDA of $\mathrm{D}=11$ supergravity, when the exterior $p$-forms are parametrized in terms of the hidden 1-forms $B_{a b}, B_{a_{1} \cdots a_{5}}, \eta$. In particular, we would like to investigate the conditions under which the gauge invariance of the FDA is realized once $A^{(3)}$ is expressed in terms of hidden 1-forms. It is useful to first recall shortly the standard procedure for the construction of a minimal $\mathrm{FDA}^{8}$ starting from an ordinary (super)Lie Algebra.

Let us denote by $\sigma^{\Lambda}$ the Maurer-Cartan 1-forms of the Lie algebra, and let us construct the so-called $(p+1)$-cochains $\Omega^{i \mid(p+1)}$ in some representation $D_{j}^{i}$ of the Lie group, that is

\footnotetext{
${ }^{7}$ The closure of the superalgebra under (super)- Jacobi identities is a consequence of the $d^{2}$-closure of the Maurer-Cartan 1-forms equations.

${ }^{8} \mathrm{~A}$ minimal FDA is one where the differential of any $p$-form does not contain forms of degree greater than $p$.
} 
$(p+1)$-forms of the type:

$$
\Omega^{i \mid(p+1)}=\Omega_{\Lambda_{1} \ldots \Lambda_{p+1}}^{i} \sigma^{\Lambda_{1}} \wedge \cdots \wedge \sigma^{\Lambda_{p+1}}
$$

where $\Omega_{\Lambda_{1} \ldots \Lambda_{p+1}}^{i}$ is a constant tensor. If the given cochains are cocycles, that is if they are closed, but not exact, they are elements of the Chevalley-Eilenberg (CE in the following) Lie algebra cohomology.

When this happens, we can introduce a $p$-form $A^{i \mid(p)}$ and write the following new closed equation:

$$
d A^{i \mid(p)}+\Omega^{i \mid(p+1)}=0
$$

which, together with the Maurer-Cartan equation of the Lie Algebra, is the first germ of a FDA, containing, besides the $\sigma^{\Lambda}$, also the new $p$-form $A^{i \mid(p)}$.

The procedure can be now iterated taking as basis of new cochains $\Omega^{j \mid\left(p^{\prime}+1\right)}$ the full set of forms, namely $\sigma^{\Lambda_{i}}$ and $A^{(p)}$, and look again for cocycles. If a new cocycle $\Omega^{j \mid\left(p^{\prime}+1\right)}$ exists, then we can add again to the FDA a new equation

$$
d A^{\left(p^{\prime}\right)}+\Omega^{j \mid\left(p^{\prime}+1\right)}=0 .
$$

The procedure can again be iterated till no more cocycles can be found, obtaining in this way the largest FDA associated with the initial Lie algebra.

The extension of this procedure to Lie superalgebras is straightforward. Actually, in the supersymmetric case a set of non-trivial cocycles is generally present in superspace, due to the existence of Fierz identities obeyed by the wedge products of gravitino 1-forms. In the case of supersymmetric theories, the 1-form fields of the superalgebra one starts with are the vielbein $V^{a}$, the gravitino $\Psi$, the spin connection $\omega^{a b}$ and possibly a set of gauge fields. However one should further impose the physical request that the FDA should be described in term of fields living in ordinary superspace, whose cotangent space is spanned by the supervielbein $\left\{V^{a}, \Psi\right\}$, dual to supertranslations. This corresponds to the physical request that the super Lie algebra has a fiber bundle structure, whose base space is spanned by the supervielbein, the rest of the fields spanning a fiber $\mathcal{H}$. This in turn implies an horizontality condition on the FDA, corresponding to gauge invariance: the gauge fields belonging to $\mathcal{H}$ must be excluded from the construction of the cochains. In geometrical terms, this corresponds to require that the CE-cohomology be restricted to the so-called $\mathcal{H}$-relative CE-cohomology.

In the case of $D=11$ supergravity, one easily recognizes that the first step of the construction outlined above is the introduction of the $\mathcal{H}$-relative 4-cocycle $\frac{1}{2} \bar{\Psi} \wedge \Gamma_{a b} \Psi \wedge$ $V^{a} \wedge V^{b}$, which allows to define the 3 -form $A^{(3)}$ of the FDA satisfying

$$
d A^{(3)}=\frac{1}{2} \bar{\Psi} \wedge \Gamma_{a b} \Psi \wedge V^{a} \wedge V^{b},
$$

that is eq. (2.4). Including the new 3 -form $A^{(3)}$ in the basis of the relative cohomology of the supersymmetric FDA, we can perform the second step and construct a new cocycle of order seven, $15 A^{(3)} \wedge d A^{(3)}+\frac{\mathrm{i}}{2} \bar{\Psi} \wedge \Gamma_{a_{1} \cdots a_{5}} \Psi \wedge V^{a_{1}} \wedge \cdots V^{a_{5}}$, allowing the introduction of the 6 -form $B^{(6)}$, satisfying:

$$
d B^{(6)}=15 A^{(3)} \wedge d A^{(3)}+\frac{\mathrm{i}}{2} \bar{\Psi} \wedge \Gamma_{a_{1} \cdots a_{5}} \Psi \wedge V^{a_{1}} \wedge \cdots V^{a_{5}},
$$


that is eq. (2.5). The fact that the two cochains (3.4) and (3.5) are indeed cocycles is due to Fierz identities in $D=11$, as reported in appendix D. The second step defined above requires to enlarge the CE-relative cohomolgy to include the 3 -form $A^{(3)}$. We further remark that the inclusion of a new p-form, which is a gauge potential enjoying a gauge freedom, in the basis of the $\mathcal{H}$-relative $\mathrm{CE}$-cohomology of the FDA, is physically meaningful only if the whole of the FDA is gauge invariant. This in particular requires that the nonphysical degrees of freedom in $A^{(3)}$ and $B^{(6)}$ are projected out from the FDA.

Let us turn now to the supersymmetric FDA of $\mathrm{D}=11$ supergravity, once it is parametrized in terms of 1-forms. Now the symmetry structure is based on the hidden supergroup manifold $G$ which extends the super-Poincaré Lie group to include the extra hidden directions associated with the higher $p$-forms. We note that the procedure introduced in [6] and reviewed in section 2 (see also [37]) can be thought of as the reverse of the costruction of a FDA from a given Lie superalgebra just recalled. Indeed, one starts from the physical FDA as given a priori and tries to reconstruct, using the procedure of [6], the hidden Lie superalgebra $\mathbb{G}$ that could have originated it using the algorithm of the CE-cohomology just described.

The hidden supergroup $G$ has the structure of a principal fiber bundle $(G / \mathcal{H}, \mathcal{H})$, where $G / \mathcal{H}$ corresponds to superspace, the fiber $\mathcal{H}$ now including, besides the Lorentz transformations, also the hidden generators. More explicitly, let us rewrite the hidden Lie superalgebra $\mathbb{G}$ of $G$ as $\mathbb{G}=\mathcal{H}+\mathbb{K}$, and decompose $\mathcal{H}=H_{0}+H_{b}+H_{f}$, so that the generators $T_{\Lambda} \in \mathbb{G}$ are grouped into $\left\{J_{a b}\right\} \in H_{0},\left\{Z^{a b} ; Z^{a_{1} \cdots a_{5}}\right\} \in H_{b},\left\{Q^{\prime}\right\} \in H_{f}$ and $\left\{P_{a} ; Q\right\} \in \mathbb{K} .{ }^{9}$ We note that the subalgebra $H_{b}+H_{f}$ defines an abelian ideal of $\mathbb{G}$.

The physical condition that the CE-cohomology be restricted to the $\mathcal{H}$-relative CEcohomology corresponds now to the request that the FDA be described in term of 1-form fields living on $G / \mathcal{H}$, and this in turn implies that the hidden 1-forms in $H_{b}$ and $H_{f}$, necessary for the parametrization of $A^{(3)}$ in terms of 1-forms, do not appear in $d A^{(3)}$ (see eq. (3.4)). Actually, as we shall see, the presence of the spinor 1-form $\eta$ is exactly what makes it possible to express $d A^{(3)}$ in terms of the relative cohomology only, that is in terms of the supervielbein.

\subsection{Gauge transformations from the hidden supergroup manifold}

Taking into account the discussion above, we now consider in detail the relation between the FDA gauge transformations and those of its hidden supergroup $G$. The supersymmetric FDA, given in eq.s (2.1)-(2.5), is left invariant under the gauge transformations

$$
\left\{\begin{array}{l}
\delta A^{(3)}=d \Lambda^{(2)} \\
\delta B^{(6)}=d \Lambda^{(5)}+\frac{15}{2} \Lambda^{(2)} \wedge \bar{\Psi} \wedge \Gamma_{a b} \Psi \wedge V^{a} \wedge V^{b}
\end{array}\right.
$$

generated by the arbitrary forms $\Lambda^{(2)}$ and $\Lambda^{(5)}$.

\footnotetext{
${ }^{9}$ Here and in the following with an abuse of notation we will use, for the cotangent space of the group manifold $G$, spanned by the 1 -forms $\sigma^{\Lambda}$, the same symbols defined above for the tangent space of $G$, spanned by the vector fields $T_{\Lambda}$.
} 
The bosonic hidden 1-forms in $H_{b}$ are abelian gauge fields, whose gauge transformations are:

$$
\left\{\begin{array}{l}
\delta_{b} B_{a b}=d \Lambda_{a b} \\
\delta_{b} B_{a_{1} \cdots a_{5}}=d \Lambda_{a_{1} \cdots a_{5}}
\end{array},\right.
$$

$\Lambda^{a b}$ and $\Lambda^{a_{1} \cdots a_{5}}$ being arbitrary Lorentz-valued scalar functions.

Requiring that $A^{(3)}$, parametrized in terms of 1-forms, transforms as (3.6) under the gauge transformations (3.7) of the 1-forms, implies the gauge transformation of $\eta$ to be:

$$
\delta_{b} \eta=-E_{2} \Lambda_{a b} \Gamma^{a b} \psi-\mathrm{i} E_{3} \Lambda_{a_{1} \cdots a_{5}} \Gamma^{a_{1} \cdots a_{5}} \psi,
$$

consistently with the condition $D \delta \eta=\delta D \eta$.

In this case the corresponding 2 -form gauge parameter of $A^{(3)}$ turns out to be:

$$
\begin{aligned}
\Lambda^{(2)}= & T_{0} \Lambda_{a b} V^{a} \wedge V^{b}+3 T_{1} \Lambda_{a b} B_{c}^{b} \wedge B^{c a} \\
& +T_{2}\left(2 \Lambda_{b_{1} a_{1} \ldots a_{4}} B_{b_{2}}^{b_{1}} \wedge B^{b_{2} a_{1} \ldots a_{4}}-B_{b_{1} a_{1} \ldots a_{4}} \Lambda_{b_{2}}^{b_{1}} \wedge B^{b_{2} a_{1} \ldots a_{4}}\right) \\
& +2 T_{3} \epsilon_{a_{1} \ldots a_{5} b_{1} \ldots b_{5} m} \Lambda^{a_{1} \ldots a_{5}} \wedge B^{b_{1} \ldots b_{5}} \wedge V^{m} \\
& +3 T_{4} \epsilon_{m_{1} \ldots m_{6} n_{1} \ldots n_{5}} \Lambda^{m_{1} m_{2} m_{3} p_{1} p_{2}} \wedge B^{m_{4} m_{5} m_{6} p_{1} p_{2}} \wedge B^{n_{1} \ldots n_{5}} \\
& +S_{2} \bar{\Psi} \wedge \Gamma^{a b} \eta \Lambda_{a b}+\mathrm{i} S_{3} \bar{\Psi} \wedge \Gamma^{a_{1} \ldots a_{5}} \eta \Lambda_{a_{1} \ldots a_{5}} .
\end{aligned}
$$

Considering also the gauge transformation of the spinor 1-form $\eta$ generated by the tangent vector in $H_{f}$, we have

$$
\delta \eta=D \varepsilon^{\prime}+\delta_{b} \eta
$$

where we have introduced the infinitesimal spinor parameter $\varepsilon^{\prime}$. The 2-form gauge parameter $\tilde{\Lambda}^{(2)}$ corresponding to the transformation in $H_{f}$ is then:

$$
\tilde{\Lambda}^{(2)}=-\mathrm{i} S_{1} \bar{\Psi} \wedge \Gamma_{a} \varepsilon^{\prime} V^{a}-S_{2} \bar{\Psi} \wedge \Gamma^{a b} \varepsilon^{\prime} B_{a b}-\mathrm{i} S_{3} \bar{\Psi} \wedge \Gamma^{a_{1} \ldots a_{5}} \varepsilon^{\prime} B_{a_{1} \ldots a_{5}} .
$$

In the following we are going to show that all the diffeomorfisms in the hidden supergroup $G$, generated by Lie derivatives, are invariances of the FDA, the ones in the fiber $\mathcal{H}$ directions being associated with a particular form of the gauge parameters of the FDA gauge transformations (3.6).

Let us first show that eq. (3.9) can be rewritten in a rather simple way using the contraction operator in the hidden Lie superalgebra $\mathbb{G}$ of $G$. Defining the tangent vector:

$$
\vec{z} \equiv \Lambda_{a b} Z^{a b}+\Lambda_{a_{1} \cdots a_{5}} Z^{a_{1} \cdots a_{5}} \in H_{b}
$$

one finds that a gauge transformation leaving invariant the $D=11 \mathrm{FDA}$ is recovered, once $A^{(3)}$ is parametrized in terms of 1 -forms, if:

$$
\Lambda^{(2)}=\imath_{\vec{z}}\left(A^{(3)}\right)
$$

where $\imath$ denotes the contraction operator. This result is actually true as a consequence of the set of relations (A.1) obeyed by the coefficients of the parametrization (2.9), that is under 
the same conditions required by supersymmetry for the consistency of the parametrization (2.9). Introducing the Lie derivative $\ell_{\vec{z}} \equiv d \imath_{\vec{z}}+\imath_{\vec{z}} d$, we find the corresponding gauge transformation of $A^{(3)}$ to be:

$$
\delta A^{(3)}=d\left(\imath_{\vec{z}}\left(A^{(3)}\right)\right)=\ell_{\vec{z}} A^{(3)} .
$$

The last equality follows since $d A^{(3)}$, as given in (2.4), is invariant under transformations generated by $\vec{z}$ corresponding to the gauge invariance of the supervielbein. Note that this is in agreement with the fact that the right hand side of $d A^{(3)}$ is in the relative $\mathcal{H} \mathrm{CE}$ cohomology .

To recover the general gauge transformation of $B^{(6)}$ in terms of the hidden algebra would require the knowledge of its explicit parametrization in terms of 1-forms, which at the moment we ignore. ${ }^{10}$ However, if we assume that its behavior under gauge transformations be still generated by $\vec{z}$ through Lie derivatives, just like for $A^{(3)}$, namely if we require:

$$
\Lambda^{(5)}=\imath_{\vec{z}}\left(B^{(6)}\right)
$$

where $B^{(6)}$ is intended as parametrized in terms of 1 -forms in $\mathbb{G}$, then a straightforward computation gives:

$$
\begin{aligned}
\delta B^{(6)} & =\ell_{\vec{z}} B^{(6)}=d\left(\imath_{\vec{z}}\left(B^{(6)}\right)\right)+\iota_{\vec{z}}\left(d B^{(6)}\right)=d \Lambda^{(5)}+\imath_{\vec{z}}\left(15 A^{(3)} \wedge d A^{(3)}\right) \\
& =d \Lambda^{(5)}+15 \Lambda^{(2)} \wedge d A^{(3)}
\end{aligned}
$$

which indeed reproduces eq. (3.6). The assumption (3.15) is corroborated by the analogous computation in the seven dimensional model considered in section 4 . In that case we can use, together with that of $B^{(3)}$, the explicit parametrization of the Hodge dual related $B^{(2)}$ appearing in the dimensional reduction of the eleven dimensional 6 -form $B^{(6)}$. As we shall see the assumption (3.15) can be fully justified if we think of $B^{(2)}$ as a remnant of $B^{(6)}$ in the dimensional reduction.

We stress that the gauge transformations (3.14) and (3.16) are not fully general, since the corresponding gauge parameters are not fully general, being they restricted to the ones satisfying (3.13), (3.15).

We should further still consider the gauge transformations generated by the other elements of $\mathcal{H}$. Since the Lorentz transformations, belonging to $H_{0} \subset \mathcal{H}$, are not effective on the FDA, all the higher $p$-forms being Lorentz-invariant, this analysis reduces to consider the transformations induced by the tangent vector $Q^{\prime} \in H_{f} \subset \mathcal{H}$. Let us then consider:

$$
\vec{q} \equiv \vec{\varepsilon}^{\prime} Q^{\prime} \in H_{f} .
$$

We find $\delta_{\vec{q}} \eta=D \varepsilon^{\prime}=\ell_{\vec{q}} \eta$ and:

$$
\begin{aligned}
\delta_{\vec{q}} A^{(3)} & =-\mathrm{i} S_{1} \bar{\Psi} \wedge \Gamma_{a} D \varepsilon^{\prime} V^{a}-S_{2} \bar{\Psi} \wedge \Gamma^{a b} D \varepsilon^{\prime} B_{a b}-\mathrm{i} S_{3} \bar{\Psi} \wedge \Gamma^{a_{1} \cdots a_{5}} D \varepsilon^{\prime} B_{a_{1} \cdots a_{5}} \\
& =d \imath_{\vec{q}} A^{(3)}=\ell_{\vec{q}} A^{(3)}
\end{aligned}
$$

\footnotetext{
${ }^{10}$ Work is in progress on this topic.
} 
where in the second line, after integration by parts, we used the relation on the $S_{i}$ :

$$
S_{1}+10 S_{2}-720 S_{3}=0
$$

following from 3-gravitino Fierz identities (see appendix D). Note that indeed eq. (3.18) reproduces $D \tilde{\Lambda}^{(2)}$, in terms of $\tilde{\Lambda}^{(2)}$ defined in eq. (3.11).

\subsection{The role of the nilpotent fermionic generator $Q^{\prime}$}

In deriving the gauge transformations leaving invariant the supersymmetric FDA, in terms of hidden 1-forms, a crucial role is played by the spinor 1-form $\eta$ dual to the nilpotent generator $Q^{\prime} \in H_{f}$. Indeed, besides the fact that it is required for the closure of the hidden superalgebra $\mathbb{G}$, it also guarantees the gauge invariance of the FDA, because of its non trivial gauge transformation, given in eq. (3.8).

Actually, we may think of the spinor 1-form $\eta$ as playing the role of an intertwining field between the base superspace and the fiber $\mathcal{H}$ of the principal fiber bundle corresponding to the hidden supergroup manifold $G=\{G / \mathcal{H}, \mathcal{H}\}$. This is also evident from its covariant differential $D \eta$, eq. (2.8), which is parametrized not only in terms of the supervielbein, as it happens for all the fields of the FDA and for $D B_{a b}$ and $D B_{a_{1} \cdots a_{5}}$, eq. (2.7), but also in terms of the gauge fields in $H_{b}$, see eq. (2.17). In the following, we are going to clarify the role of $\eta$ in the more general context of the construction of FDAs discussed above, showing that its presence is essential to have a well defined, gauge invariant supersymmetric FDA.

A clarifying example corresponds to considering a singular limit where $\eta$ is set equal to zero, so that its dual generator $Q^{\prime}$ can be dropped out from $\mathbb{G}$. This limit may be obtained, in its simplest form, by redefining the coefficients (2.11) appearing in the parametrization of $A^{(3)}$ as follows:

$$
E_{2} \rightarrow E_{2}^{\prime}=\epsilon E_{2}, \quad E_{3} \rightarrow E_{3}^{\prime}=\epsilon^{2} E_{3}
$$

and then taking the limit $\epsilon \rightarrow 0$. One finds:

$$
T_{0} \rightarrow \tilde{T}_{0}=\frac{1}{6}, \quad T_{1} \rightarrow \tilde{T}_{1}=-\frac{1}{90}, \quad T_{2}=T_{3}=T_{4} \rightarrow 0, \quad E_{1}=E_{2}=E_{3} \rightarrow 0,
$$

while $S_{1}, S_{2}, S_{3} \rightarrow \infty$ in the limit. Recalling the parametrization of $A^{(3)},(2.9)$, we see that setting $\eta=0$, the following finite limit can be obtained for $A^{(3)}$ :

$$
A^{(3)} \rightarrow A_{\lim }^{(3)}=\tilde{T}_{0} B_{a b} \wedge V^{a} \wedge V^{b}+\tilde{T}_{1} B_{a b} \wedge B_{c}^{b} \wedge B^{c a} .
$$

so that its differential gives:

$$
d A_{\lim }^{(3)}=\tilde{T}_{0}\left(\frac{1}{2} \bar{\Psi} \Gamma_{a b} \Psi \wedge V^{a} \wedge V^{b}-\mathrm{i} B_{a b} \wedge \bar{\Psi} \Gamma^{a} \Psi \wedge V^{b}\right)+\frac{3}{2} \tilde{T}_{1} \bar{\Psi} \Gamma_{a b} \wedge \Psi \wedge B_{c}^{b} \wedge B^{c a} .
$$

We see that the parametrization (3.22) does not reproduce the FDA (2.4), being in fact obtained by a singular limit. However this different FDA is based on the same hidden algebra $\mathbb{G}$, where now the cocycles are in the $H_{0}$-relative $\mathrm{CE}$ cohomology. Indeed $d A_{\text {lim }}^{(3)}$ is now expanded on a basis of the enlarged superspace $K_{\text {enlarged }}=K+H_{b}$, which includes, besides the supervielbein, also the bosonic hidden 1-forms. The case where all the $E_{i}$ are 
proportional to the same power of $\epsilon$ can be done on the same lines, it again requires $\eta=0$ and leads to an $A_{\text {lim }}^{(3)}$ with all $\tilde{T}_{i} \neq 0$ (for $i=0,1, \cdots 4$ ). In this case $d A_{\text {lim }}^{(3)}$ is expanded on a basis of the $K_{\text {enlarged }}$ also including $B_{a_{1} \cdots a_{5}}$.

A singular limit of the parametrization of $A^{(3)}$ was already considered in [16, 17]. The limit considered in $[16,17]$ is similar to ours (where our parameters $E_{i}$ play a role similar to their parameter $s) .{ }^{11}$ There, the authors were studying the description of the hidden superalgebra as an expansion of $\operatorname{OSp}(1 \mid 32)$. They observed that a singular limit exists (which includes ours as a special case) such that the authomorphism group of the FDA is enlarged from what we called $\mathcal{H}$ to $\operatorname{Sp}(32)$, but where the trivialization of the FDA in terms of an explicit $A^{(3)}$, written in terms of 1 -forms, breaks down. From the above analysis we see that, at least for the restriction of the limit considered here, what does break down is in fact the trivialization of the FDA on ordinary superspace, while a trivialization on $K_{\text {enlarged }}$ is still possible.

Note, however, that in this case the gauge invariance of the new FDA requires that $B_{a b}$ (and analogously $B_{a_{1} \cdots a_{5}}$ ) is not a gauge field anymore. Correspondingly, $A_{\text {lim }}^{(3)}$ does not enjoy gauge freedom, all of its degrees of freedom propagating in $K_{\text {enlarged }}$. It may then be interpreted as a gauge-fixed form of $A^{(3)}$. Indeed, it is precisely the gauge transformation of $\eta$, given in eq. (3.8), that guarantees the gauge transformation of $A^{(3)}$ to be (3.6). Actually, this relies on the fact that $D \eta \in K_{\text {enlarged }}$ as we already observed previously, when we introduced eq. (3.8). Note that the transformation (3.7), even if it is not a gauge transformation in this limit case, still generates a diffeomorphism leaving invariant the new FDA (which is indeed based on the same supergroup $G$ ), since

$$
\delta_{\vec{z}} A_{\mathrm{lim}}^{(3)}=\ell_{\vec{z}} A_{\mathrm{lim}}^{(3)} .
$$

A gauge transformation bringing $A^{(3)}$ to $A_{\mathrm{lim}}^{(3)}$ and, more generally, a gauge transformation such that $\eta^{\prime}=\eta+\delta \eta=0$, is associated with transformations (3.10), generated by the tangent vector $\vec{q}$ introduced in (3.17), in the particular case $\delta_{\vec{q}} \eta=D \varepsilon^{\prime}=-\eta$.

In conclusion, the role of the extra fermionic nilpotent generator amounts to require the hidden 1-forms of the Lie superalgebra to be true gauge fields living on the fiber $\mathcal{H}$ of the associated principal fiber bundle $\{G / \mathcal{H}, \mathcal{H}\} .{ }^{12}$ It plays a role similar to a BRST ghost, since it guarantees that only the physical degrees of freedom of the exterior forms appear in the supersymmetric FDA in a "dynamical" way: this amounts to say that, once the superspace is enlarged to $K_{\text {enlarged }}$, in the presence of $\eta$ and more generally of a non empty $H_{f}$, no explicit constraint has to be imposed on the fields, since the non-physical degrees of freedom of the fields in $H_{b}$ and in $H_{f}$ transform into each other and do not contribute to the FDA.

\section{The hidden gauge algebra of $D=7, \mathcal{N}=2$ Free Differential Algebra}

The same procedure explained in the eleven dimensional case can be applied to lower dimensional supergravity theories, in order to associate with any such theory containing

\footnotetext{
${ }^{11}$ More precisely, the singular limit considered in $[16,17]$ is given in terms of a parmater $s \rightarrow 0$. The relation between their and our parameters is $s \propto E_{2}-60 E_{3}$. See also footnote 6 .

${ }^{12}$ Note that this is equivalent to require that the construction of the FDA from Lie algebra of the supergroup $G$ be done using the $\mathcal{H}$-relative CE cohomology of $\mathbb{G}$.
} 
$p$-forms (with $p>1$ ), a hidden Lie superalgebra containing, as a subalgebra, the superPoincaré algebra. Since in the $\mathrm{D}=11$ theory the closure of the FDA and of the corresponding hidden superalgebra are strictly related to 3-gravitino Fierz identities of the given theory, the same must happen in any lower dimensions.

As an interesting example we consider in this section the minimal $D=7, \mathcal{N}=2$ theory (not coupled to matter), where the hidden structure turns out to be particularly rich since, as we will see, in its most general form it includes two nilpotent fermionic generators.

Working as in the eleven dimensional case within the geometric formulation of superspace $p$-forms, its physical content on space-time is given by the vielbein 1-form $V^{a}$, a triplet of vectors 1 -forms $A^{x}(x=1,2,3)$, a 2-form $B^{(2)}$, together with a gravitino 1-form Dirac spinor which we describe as a couple of 8-component spin-3/2 pseudo-Majorana fields $\psi_{A \mu}(A=1,2)$ satisfying the reality condition $\bar{\psi}^{A}=\epsilon^{A B}\left(\psi_{B}\right)^{T} \cdot{ }^{13}$

The interacting $D=7$ minimal theory was studied, at the lagrangian level, by many authors [38]-[41]. In particular, in [38] it was observed that one can trade the 2-form formulation of the theory by a formulation in terms of a 3 -form, $B^{(3)}$, the two being related by Hodge-duality of the corresponding field strengths on space-time, and they give rise to different lagrangians. From our point of view, where only the FDA is considered (and not a Lagrangian description), both forms are required for a fully general formulation, together with a triplet of 4-forms, $A^{x \mid(4)}$, whose field strengths are Hodge-dual to the gauge vectors $A^{x}$.

One of the main reasons for choosing the minimal $D=7$ model is related to the fact that in this case we will be able to find an explicit parametrization in terms of 1-forms of both $B^{(2)}$ and $B^{(3)}$, whose space-time field strengths are related by Hodge duality. We will find that in this case a general parametrization requires the presence of two independent hidden spinor 1 -forms. Since $B^{(2)}$ in $D=7$ can be obtained by dimensional reduction of $B^{(6)}$ in the eleven dimensional FDA, this investigation also allows to shed some light on the extension of the hidden superalgebra of $D=11$ supergravity when also the parametrization of $B^{(6)}$, still unknown, would be considered (see section 5).

The minimal $N=2, D=7$ supergravity is based on the following supersymmetric FDA:

$$
\begin{aligned}
R^{a b} & \equiv d \omega^{a b}-\omega^{a}{ }_{c} \wedge \omega^{c b}=0, \\
T^{a} & \equiv D V^{a}-\frac{\mathrm{i}}{2} \bar{\psi}^{A} \wedge \Gamma^{a} \psi_{A}=0, \\
\rho & \equiv D \psi=0, \\
F^{x} & \equiv d A^{x}-\frac{\mathrm{i}}{2} \sigma^{x \mid B} \bar{\psi}^{A} \wedge \psi_{B}=0, \\
F^{(3)} & \equiv d B^{(2)}+d A^{x} \wedge A^{x}-\frac{\mathrm{i}}{2} \bar{\psi}^{A} \wedge \Gamma_{a} \psi_{A} \wedge V^{a}=0, \\
G^{(4)} & \equiv d B^{(3)}-\frac{1}{2} \bar{\psi}^{A} \wedge \Gamma_{a b} \psi_{A} \wedge V^{a} \wedge V^{b}=0,
\end{aligned}
$$

\footnotetext{
${ }^{13}$ The charge conjugation matrix in $\mathrm{D}=7$ can always be chosen $C=\mathbb{1}$.
} 


$$
\begin{aligned}
F^{x(4)} \equiv & d A^{x \mid(4)}+\frac{1}{2}\left(d A^{x} \wedge B^{(3)}-A^{x} \wedge d B^{(3)}\right) \\
& -\frac{1}{6} \sigma_{A}^{x \mid B} \bar{\psi}^{A} \wedge \Gamma_{a b c} \psi_{B} \wedge V^{a} \wedge V^{b} \wedge V^{c}=0,
\end{aligned}
$$

where now $D$ denotes the $D=7$ Lorentz-covariant differential and $\sigma_{A}^{x \mid B}$ are the usual Pauli matrices. As already mentioned the $d^{2}$-closure of this FDA relies on the Fierz identities relating gravitino 3 - and 4 -forms currents in $D=7$.

To find the hidden superalgebra, let us introduce the following set of bosonic Lorentzindexed 1-forms: $B_{a}$, associated with $B^{(2)}, B_{a b}$, associated with $B^{(3)}, A_{a b c}^{x}$, associated with $A^{x \mid 4}$, requiring their Maurer-Cartan equations to be:

$$
\begin{aligned}
D B_{a b} & =\alpha \bar{\psi}^{A} \wedge \Gamma_{a b} \psi_{A}, \\
D B_{a} & =\beta \bar{\psi}^{A} \wedge \Gamma_{a} \psi_{A}, \\
D A_{a b c}^{x \mid} & =\gamma \sigma_{A}^{x \mid B} \bar{\psi}^{A} \wedge \Gamma_{a b c} \psi_{B} .
\end{aligned}
$$

whose integrability conditions are automatically satisfied since $R^{a b}=0$. The arbitrary choice of the coefficients in the right-hand-side fixes the normalization of the bosonic 1forms $B_{a}, B_{a b}$ and $A_{a b c}^{x \mid}$. In the following, we will choose $\alpha=\frac{1}{2}, \beta=\frac{i}{2}, \gamma=\frac{1}{6}$.

The bosonic forms $B^{(2)}$ and $B^{(3)}$ will be parametrized, besides the 1-forms $V^{a}, A^{x}$ already present in the FDA, also in terms of the new 1-forms $B_{a}, B_{a b}, A^{x \mid}{ }_{a b c}$, and as we are going to show, the consistency of their parametrizations also requires the presence of two nilpotent fermionic 1-forms, $\eta_{A}$ in the parametrization of $B^{(2)}$ and $\xi_{A}$ in the one of $B^{(3)}$, whose covariant derivatives satisfy:

$$
\begin{aligned}
D \eta_{A}= & l_{1} \Gamma_{a} \psi_{A} \wedge V^{a}+l_{2} \Gamma^{a} \psi_{A} \wedge B_{a}+l_{3} \Gamma^{a b} \psi_{A} \wedge B_{a b} \\
& +l_{4} \psi_{B} \sigma_{A}^{x \mid B} \wedge A^{x}+l_{5} \Gamma^{a b c} \psi_{B} \sigma^{x \mid B}{ }_{A} \wedge A^{x \mid}{ }_{a b c} \\
D \xi_{A}= & e_{1} \Gamma_{a} \psi_{A} \wedge V^{a}+e_{2} \Gamma^{a} \psi_{A} \wedge B_{a}+e_{3} \Gamma^{a b} \psi_{A} \wedge B_{a b} \\
& +e_{4} \psi_{B} \sigma_{A}^{x \mid B} \wedge A^{x}+e_{5} \Gamma^{a b c} \psi_{B} \sigma_{A}^{x \mid B} \wedge A_{a b c}^{x \mid}
\end{aligned}
$$

where $l_{i}, e_{i}$ are so far unspecified structure constants of the hidden superalgebra, constrained to satisfy (from the integrability of $D \eta_{A}$ and $D \xi_{A}$ and use of the Fierz identities):

$$
\begin{aligned}
-\mathrm{i} l_{1}-\mathrm{i} l_{2}+6 l_{3}-\mathrm{i} l_{4}-10 l_{5} & =0 \\
-\mathrm{i} e_{1}-\mathrm{i} e_{2}+6 e_{3}-\mathrm{i} e_{4}-10 e_{5} & =0 .
\end{aligned}
$$

The consistency of the parametrizations amounts to require that the differential of $B^{(2)}$ and $B^{(3)}$, as given in equations (4.5) and (4.6), must be reproduced by the differential of their parametrizations (4.13), (4.14). This is analogous to what happens in $D=11$; in that case, however, only the parametrization of the 3 -form was considered, and its closure, besides the precise values of the coefficients, required the presence of just one spinor 1-form dual to a nilpotent fermionic generator. 
Explicitly we give the following general Ansatz for the parametrization of $B^{(2)}$ and $B^{(3)}$ in terms of the 1 -forms $\left\{V^{a}, \psi_{A}, B_{a}, B_{a b}, A^{x \mid}{ }_{a b c}, \xi_{A}, \eta_{A}\right\}:^{14}$

$$
\begin{aligned}
B^{(2)}= & \sigma B_{a} \wedge V^{a}+\tau \bar{\psi}^{A} \wedge \eta_{A}, \\
B^{(3)}= & \tau_{0} B_{a b} \wedge V^{a} \wedge V^{b}+\tau_{1} B_{a b} \wedge B^{a} V^{b}+\tau_{2} B_{a b} \wedge B^{a} B^{b}+\tau_{3} B_{a b} \wedge B^{b c} \wedge B_{c}^{a} \\
& +\epsilon_{a b_{1} \ldots b_{3} c_{1} \ldots c_{3}}\left(\tau_{4} V^{a}+\tau_{5} B^{a}\right) \wedge A^{x \mid b_{1} \ldots b_{3}} \wedge A_{c_{1} \ldots c_{3}}^{x} \\
& +\tau_{6} B_{a b} \wedge A_{a c d}^{x} \wedge A^{x \mid b c d}+\tau_{7} \epsilon_{x y z} A^{x} \wedge A_{a b c}^{y} \wedge A^{z \mid a b c} \\
& +\tau_{8} \epsilon_{x y z} A^{x} \wedge A^{y} \wedge A^{z}+\tau_{9} \epsilon_{x y z} \epsilon_{a b c d l m n} A^{x \mid a b c} \wedge A^{y \mid d l p} \wedge A_{p}^{z \mid m n}{ }_{p} \\
& +\sigma_{1} \bar{\psi}^{A} \wedge \Gamma_{a} \xi_{A} \wedge V^{a}+\sigma_{2} \bar{\psi}^{A} \wedge \Gamma_{a} \xi_{A} \wedge B^{a}+\sigma_{3} \bar{\psi}^{A} \wedge \Gamma_{a b} \xi_{A} \wedge B^{a b} \\
& +\sigma_{4} \bar{\psi}^{A} \wedge \xi_{B} \sigma_{A}^{x \mid B} \wedge A^{x}+\sigma_{5} \bar{\psi}^{A} \wedge \Gamma^{a b c} \xi_{B} \sigma_{A}^{x \mid B} \wedge A_{a b c}^{x \mid} .
\end{aligned}
$$

The set of coefficients $\left\{\tau_{j}\right\},\left\{\sigma_{i}\right\}$ are determined by requiring that the parametrizations (4.13) and (4.14) satisfy the FDA, in particular eq.s (4.5), (4.6). Their explicit expression is given in appendix B. However, we still have the freedom to fix the normalization of the spinor 1-forms $\xi_{A}, \eta_{A}$. We are going to fix them in order to obtain a simple expression. In particular we choose the normalization of $\eta_{A}$ by imposing, in the parametrization of $B^{(2)}, \tau=1$. As far as the normalization of $\xi_{A}$ is concerned, using the general solution for the coefficients given in appendix $\mathrm{B}$, we find $\frac{e_{2}}{\sigma_{2}}=\frac{e_{5}}{\sigma_{5}} \equiv H$, where, with the normalization chosen for the bosonic 1-forms:

$$
H=-2\left(e_{1}+e_{2}-2 \mathrm{i} e_{3}\right)\left(e_{1}+e_{2}-2 \mathrm{i} e_{5}\right) .
$$

We choose $H=1$, which is a valid normalization in all cases where $H \neq 0$, that is for $e_{1}+e_{2} \neq 2 \mathrm{i} e_{3}$ or $e_{1}+e_{2} \neq 2 \mathrm{i} e_{5}$. Actually the general solution for the parameters given in appendix $\mathrm{B}$ shows that to choose $\tau \neq 0, H \neq 0$ are not restrictive assumptions, since the cases $\tau=0$ and/or $H=0$ would correspond to singular limits where the gauge structure of the supersymmetric FDA breaks down. This is strictly analogous to what we discussed in section 3.2 for the $\mathrm{D}=11$ case as far as the gauge structure of the theory is concerned.

With the above normalizations we obtain:

$$
\begin{aligned}
& \sigma=2 \mathrm{i} l_{2}, \quad l_{1}=\frac{i}{2}\left(-1+2 \mathrm{i} l_{2}\right), \quad l_{4}=\frac{\mathrm{i}}{2}, \quad l_{3}=l_{5}=0, \\
& \tau_{0}=2\left[\mathrm{i} e_{1}\left(e_{3}-e_{5}\right)+\left(\frac{\mathrm{i}}{2} e_{2}+e_{3}\right)\left(\frac{\mathrm{i}}{2} e_{2}+e_{5}\right)\right] \\
& \tau_{1}=-4 \mathrm{i}_{2}\left(\mathrm{i} e_{2}+2 e_{5}\right), \quad \tau_{2}=-2 e_{2}^{2}, \quad \tau_{3}=-\frac{8}{3} e_{3}\left(e_{3}-2 e_{5}\right) \\
& \tau_{4}=e_{5}\left(\mathrm{i} e_{2}+2 e_{5}\right), \quad \tau_{5}=-\mathrm{ie}_{2} e_{5}, \quad \tau_{6}=36 e_{5}^{2} \\
& \tau_{7}=-12 e_{5}^{2} \text {, } \\
& \tau_{8}=\frac{2}{3} e_{4}\left[e_{1}+e_{2}-6 \mathrm{i}\left(e_{3}+e_{5}\right)\right], \quad \tau_{9}=-3 e_{5}^{2} \\
& \sigma_{1}=-e_{1}-2 e_{2}+4 \mathrm{i} e_{5}, \quad \sigma_{2}=e_{2}, \quad \sigma_{3}=-e_{3}+2 e_{5}, \quad \sigma_{4}=-e_{4},
\end{aligned}
$$

where the $e_{i}$ are constrained by (4.12). ${ }^{15}$

\footnotetext{
${ }^{14}$ We should in principle also consider the parametrization of the 4-form $A_{(4)}^{x}$. This deserves further investigation. Some work is in progress on this point.

${ }^{15}$ We observe that the combination $\tau_{4} V^{a}+\tau_{5} B_{a} \equiv \tilde{B}_{a}$ could be used, instead of $B_{a}$, in the parametrization of $B^{(3)}$. This redefinition simplifies the expression of $B^{(3)}$, in particular the term $B_{a b} \wedge \tilde{B}^{a} \wedge V^{b}$ vanishes.
} 


\subsection{The hidden superalgebra}

Let us write now, analogously to what was done in $D=11$, the $D=7$ hidden superalgebra in terms of generators $T_{\Lambda}$ dual to the set of 1 -forms $\sigma^{\Lambda}$ of the theory. In this case we have

$$
\sigma^{\Lambda}=\left\{V^{a}, \psi_{A}, \omega^{a b}, B_{a}, B_{a b}, A_{a b c}^{x \mid}, \xi_{A}, \eta_{A}\right\}
$$

and

$$
T_{\Lambda}=\left\{P_{a}, Q_{A}, J_{a b}, T^{x}, Z^{a}, Z^{a b}, T^{x \mid a b c}, Q_{A}^{\prime}, Q_{A}^{\prime \prime}\right\} .
$$

The only non-trivial mappings are now:

$$
\begin{aligned}
& \psi_{A}\left(Q^{B}\right)=\delta_{A}^{B}, \quad \xi_{A}\left(Q^{\prime B}\right)=\delta_{A}^{B}, \\
& \eta_{A}\left(Q^{\prime \prime B}\right)=\delta_{A}^{B}, \\
& V^{a}\left(P_{b}\right)=\delta_{b}^{a} \text {, } \\
& B_{a}\left(Z^{b}\right)=\delta_{a}^{b}, \\
& B_{a b}\left(Z^{c d}\right)=2 \delta_{a b}^{c d}, \\
& A^{x}\left(T^{y}\right)=\delta^{x y}, \\
& A_{a b c}^{x \mid}\left(T^{y \mid l m n}\right)=3 ! \delta^{x y} \delta_{a b c}^{l m n}, \\
& B_{a_{1} \cdots a_{5}}\left(Z^{b_{1} \cdots b_{5}}\right)=5 ! \delta_{a_{1} \cdots a_{5}}^{b_{1} \cdots b_{5}},
\end{aligned}
$$

so that the (anti)-commutators of the superalgebra (besides the Poincaré Lie algebra) can be written as

$$
\begin{aligned}
\left\{Q^{A}, \bar{Q}_{B}\right\} & =-\mathrm{i} \Gamma^{a}\left(P_{a}+\eta_{a b} Z^{b}\right) \delta_{B}^{A}-\frac{1}{2} \Gamma_{a b} Z^{a b} \delta_{B}^{A}-\sigma_{B}^{x \mid A}\left(\mathrm{i} T^{x}+\frac{1}{18} \Gamma^{a b c} T_{a b c}^{x}\right), \\
{\left[Q_{A}, P_{a}\right] } & =-2 \Gamma_{a}\left(e_{1} Q_{A}^{\prime}+l_{1} Q_{A}^{\prime \prime}\right), \\
{\left[Q_{A}, Z^{a}\right] } & =-2 \Gamma^{a}\left(e_{2} Q_{A}^{\prime}+l_{2} Q_{A}^{\prime \prime}\right), \\
{\left[Q_{A}, Z^{a b}\right] } & =-4 e_{3} \Gamma^{a} Q_{A}^{\prime}, \\
{\left[Q_{A}, T^{x}\right] } & =-2 \sigma_{A}^{x \mid B}{ }_{A}\left(e_{4} Q_{B}^{\prime}+l_{4} Q_{B}^{\prime \prime}\right), \\
{\left[Q_{A}, T^{x \mid a b c}\right] } & =-12 e_{5} \Gamma^{a b c} \sigma_{A}^{x \mid B} Q_{B}^{\prime}, \\
{\left[J_{a b}, Z^{c}\right] } & =-2 \delta_{[a}^{c} Z_{b]}, \\
{\left[J_{a b}, Z^{c d}\right] } & =-4 \delta_{[a}^{[c} Z_{b]}^{d]}, \\
{\left[J_{a b}, T^{x \mid c_{1} c_{2} c_{3}}\right] } & \left.=-12 \delta_{[a}^{\left[c_{1}\right.} T^{x \mid} c_{2} c_{3}\right] \\
{\left[J_{a b}, Q^{A}\right] } & =-\Gamma_{a b} Q^{A}, \\
{\left[J_{a b}, Q^{\prime A}\right] } & =-\Gamma_{a b} Q^{\prime A} .
\end{aligned}
$$

All the other possible commutators vanish.

Lagrangian subalgebras. Let us consider here two relevant subalgebras of the general hidden superalgebra presented above, where only one nilpotent spinor 1-form appears. We call them "electric hidden subalgebras" or "lagrangian subalgebras" because of their relevance for the construction of the lagrangian, as we will clarify in the following discussion.

The first subalgebra is the one where $Q_{A}^{\prime}=Q_{A}^{\prime \prime}=\frac{1}{2} \hat{Q}_{A}$. This corresponds to consider a FDA including both $B^{(2)}$ and $B^{(3)}$, where however the same nilpotent spinor 1-form appears in the parametrizations (4.13) and (4.14), namely $\eta_{A}=\xi_{A}$. In this case the Maurer-Cartan equations (4.9) and (4.10) coincide, implying $\left\{e_{i}\right\}=\left\{l_{i}\right\}$, so that in particular $e_{3}=e_{5}=0$ 
in this case, since $l_{3}=l_{5}=0$. This in turn implies, on the set of $\left\{\tau_{j}\right\}$ given in (4.17), that all the contributions in $B_{a b}$ and $A_{a b c}^{x}$ in the parametrization of $B^{(3)}$ disappear, and the corresponding generators $Z_{a b}$ and $T_{a b c}^{x}$ decouple and can be set to zero. The resulting subalgebra is:

$$
\begin{aligned}
\left\{Q^{A}, \bar{Q}_{B}\right\} & =-\mathrm{i} \Gamma_{a}\left(P^{a}+Z^{a}\right) \delta_{B}^{A}-\mathrm{i} \sigma_{B}^{x \mid A} T^{x} \\
{\left[Q_{A}, P^{a}\right] } & =-2 e_{1} \Gamma^{a} \hat{Q}_{A} \\
{\left[Q_{A}, Z^{a}\right] } & =-2 e_{2} \Gamma^{a} \hat{Q}_{A} \\
{\left[Q_{A}, T^{x}\right] } & =-2 e_{4} \sigma^{x \mid B}{ }_{A} \hat{Q}_{B} .
\end{aligned}
$$

Note that the same subalgebra can be obtained equivalently by truncating the hidden algebra to the subalgebra where $Q_{A}^{\prime} \rightarrow 0$ or, equivalently, $\xi_{A} \rightarrow 0$. However, recalling the discussion of sections 3.1,3.2 about the role of the nilpotent spinor generators for the consistency of the gauge structure of the FDA, referring in particular to the singular limit $\eta \rightarrow 0,{ }^{16}$ from the FDA point of view this corresponds to consider instead the subFDA where only $A^{x}$ and $B^{(2)}$ appear, but not their mutually non-local forms $B^{(3)}, A^{x \mid(4)}$. This is indeed the appropriate framework for a lagrangian description in terms of $B^{(2)}$, as considered for example in [39, 40].

The alternative lagrangian subalgebra is found by setting instead $Q_{A}^{\prime \prime} \rightarrow 0$, implying the vanishing of the coefficients $\left\{l_{i}\right\}$. In this case the whole parametrization of $B^{(2)}$ drops out, so that this subalgebra corresponds to consider the restricted FDA where $B^{(2)}$ is excluded, together with $A^{x \mid(4)}$. This is in fact the appropriate framework for the construction of the lagrangian in terms of $B^{(3)}$ only [38]. The 1-forms $B_{a}$ and $A_{a b c}^{x}$ could still be included in the parametrization of $B^{(3)}$ as trivial deformations, and they can be consistently decoupled by setting $e_{2}=e_{5}=0$.

Let us stress that both Lagrangian subalgebras require the truncation of the superalgebra to include only one out of the two nilpotent spinors.

The hidden superalgebra we have constructed in the present section includes all the dynamical 1-forms associated with the $D=7 \mathrm{FDA}$ once it is extended to include all couples of Hodge-dual field strengths, and in this sense it is fully general. It is larger than the one just involving the fields appearing in the Lagrangian in terms of either $B^{(2)}$ or of $B^{(3)}$ only. Actually, this is reminiscent of a well know feature of four dimensional extended supersymmetric theories: there, the central extension of the supersymmetry algebra is associated with electric and magnetic charges [8], while the electric subalgebra only involves electric charges whose associated gauge potentials appear in the lagrangian description.

Let us remark that the analysis above shows that two independent spinorial generators (associated with 2 independent nilpotent spinorial charges) are necessary if we want to include in the hidden algebra description of the FDA involving both $B^{(2)}$ and $B^{(3)}$ also the 1-forms $B_{a b}$ and $A_{a b c}^{x}$, besides the 1-forms $B_{a}$ and $A^{x}$. However, we did not consider in the above description the 1-form $B_{a_{1} \cdots a_{5}}$ associated with the non-dynamical volume form $F^{(7)}=d B^{(6)}+\cdots$. We could ask if the inclusion of such extra contributions in

\footnotetext{
${ }^{16}$ The discussion of sections $3.1,3.2$ concerned in fact the eleven dimensional theory. Analogous considerations can be worked out for the seven dimensional case, as will be shown in some detail in subsection 4.3.
} 
the parametrization of $B^{(2)}$ and $B^{(3)}$ could significantly alter the results obtained, and if it would requires the presence of extra spinorial charges. This issue is discussed in the following subsection.

\subsection{Including $B_{a_{1} \cdots a_{5}}$}

To complete the analysis of the minimal theory in $D=7$, let us further investigate the superalgebra hidden in the extension of the FDA to include the (non-dynamical) form $B^{(6)}$ associated with the volume form in seven dimensions.

It contributes to the FDA as:

$$
d B^{(6)}-15 B^{(3)} \wedge d B^{(3)}=\frac{\mathrm{i}}{2} \bar{\psi} \wedge \Gamma_{a_{1} \cdots a_{5}} \psi \wedge V^{a_{1}} \cdots \wedge V^{a_{5}},
$$

as it is evident by the dimensional reduction of the eleven dimensional 6 -form, that we will treat in section 5 . The aim is double: on one hand we would like to check how the hidden algebra gets enlarged in the presence of the extra 1-form $B_{a_{1} \cdots a_{5}}$ associated with $B^{(6)}$, and in particular if it requires the presence of one more fermionic generator; on the other hand, this analysis will turn out to be useful once we will relate, in the next section, the $\mathrm{D}=7$ theory to the $\mathrm{D}=11$ one.

Let us quote below the result. We require the covariant derivative of the spinor 1-forms to be now:

$$
\begin{aligned}
D \xi_{A}= & e_{1} \Gamma_{a} \psi_{A} \wedge V^{a}+e_{2} \Gamma^{a} \psi_{A} \wedge B_{a}+e_{3} \Gamma^{a b} \psi_{A} \wedge B_{a b} \\
& +e_{4} \psi_{B} \sigma^{x \mid B}{ }_{A} \wedge A^{x}+e_{5} \Gamma^{a b c} \psi_{B} \sigma_{A}^{x \mid B} \wedge A_{a b c}^{x \mid}+e_{6} \Gamma^{a_{1} \ldots a_{5}} \psi_{A} B_{a_{1} \ldots a_{5}}, \\
D \eta_{A}= & l_{1} \Gamma_{a} \psi_{A} \wedge V^{a}+l_{2} \Gamma^{a} \psi_{A} \wedge B_{a}+l_{3} \Gamma^{a b} \psi_{A} \wedge B_{a b} \\
& +l_{4} \psi_{B} \sigma_{A}^{x \mid B} \wedge A^{x}+l_{5} \Gamma^{a b c} \psi_{B} \sigma_{A}^{x \mid B} \wedge A_{a b c}^{x \mid}+l_{6} \Gamma^{a_{1} \ldots a_{5}} \psi_{A} B_{a_{1} \ldots a_{5}} .
\end{aligned}
$$

and, besides eq.s (4.8), we define:

$$
D B_{a_{1} \ldots a_{5}}=\frac{\mathrm{i}}{2} \bar{\psi}^{A} \wedge \Gamma_{a_{1} \ldots a_{5}} \psi_{A} .
$$

The integrability conditions of (4.37) and (4.38) give:

$$
\begin{aligned}
-\mathrm{i} l_{1}-\mathrm{i} l_{2}+6 l_{3}-\mathrm{i} l_{4}-10 l_{5}-\mathrm{i} 360 l_{6} & =0, \\
-\mathrm{i} e_{1}-\mathrm{i} e_{2}+6 e_{3}-\mathrm{i} e_{4}-10 e_{5}-\mathrm{i} 360 e_{6} & =0 .
\end{aligned}
$$

We find the following new parametrizations for $B^{(2)}$ and $B^{(3)}$ :

$$
\begin{aligned}
B^{(2)}= & B_{\text {old }}^{(2)}+\chi \epsilon_{a_{1} \ldots a_{5} a b} B^{a_{1} \ldots a_{5}} \wedge B^{a b} \\
B^{(3)}= & B_{\text {old }}^{(3)}+\tau_{10} B_{a a_{1} \ldots a_{4}} \wedge B_{b}^{a} \wedge B^{b a_{1} \ldots a_{4}}+\tau_{11} \epsilon_{a_{1} \ldots a_{5} a b} B^{a_{1} \ldots a_{5}} \wedge V^{a} \wedge V^{b} \\
& +\tau_{12} \epsilon_{a_{1} \ldots a_{5} a b} B^{a_{1} \ldots a_{5}} \wedge B^{a} \wedge V^{b}+\tau_{13} \epsilon_{a_{1} \ldots a_{5} a b} B^{a_{1} \ldots a_{5}} \wedge B^{a} \wedge B^{b} \\
& +\tau_{14} \epsilon_{a_{1} \ldots a_{5} a b} B^{a_{1} \ldots a_{5}} \wedge A_{a c d}^{x} \wedge A^{x \mid b c d}++\sigma_{6} \bar{\psi}^{A} \wedge \Gamma_{a_{1} \ldots a_{5}} \xi_{A} \wedge B^{a_{1} \ldots a_{5}}
\end{aligned}
$$

where $B_{\text {old }}^{(2)}$ and $B_{\text {old }}^{(3)}$ are given by equations (4.13) and (4.14). The values of the new set of coefficients is given in appendix B. 
The result is that the parametrization of the extended forms in terms of 1-forms is more complicated in this case, but the closure of the hidden superalgebra does not require any new spinorial 1-form generator besides $\xi_{A}$ and $\eta_{A}$.

To express the superalgebra in the dual form, it is sufficient to introduce the bosonic generator $Z^{a_{1} \cdots a_{5}}$ satisfying $B_{a_{1} \cdots a_{5}}\left(Z^{b_{1} \cdots b_{5}}\right)=5 ! \delta_{a_{1} \cdots a_{5}}^{b_{1} \cdots b_{5}}$, and we get

$$
\begin{aligned}
\left\{Q^{A}, \bar{Q}_{B}\right\}= & -\left[\mathrm{i} \Gamma^{a}\left(P_{a}+\eta_{a b} Z^{b}\right)+\frac{1}{2} \Gamma_{a b} Z^{a b}+\frac{\mathrm{i}}{5 !} \Gamma_{a_{1} \cdots a_{5}} Z^{a_{1} \cdots a_{5}}\right] \delta_{B}^{A}+ \\
& -\sigma_{B}^{x \mid A}\left(\mathrm{i} T^{x}+\frac{1}{18} \Gamma_{a b c} T^{x \mid a b c}\right), \\
{\left[Q_{A}, P_{a}\right]=} & -2 \Gamma_{a}\left(e_{1} Q_{A}^{\prime}+l_{1} Q_{A}^{\prime \prime}\right), \\
{\left[Q_{A}, Z^{a}\right]=} & -2 \Gamma^{a}\left(e_{2} Q_{A}^{\prime}+l_{2} Q_{A}^{\prime \prime}\right), \\
{\left[Q_{A}, Z^{a b}\right]=} & -4 \Gamma^{a b}\left(e_{3} Q_{A}^{\prime}+l_{3} Q_{A}^{\prime \prime}\right), \\
{\left[Q_{A}, T^{x}\right]=} & -2 \sigma_{A}^{x \mid B}\left(e_{4} Q_{B}^{\prime}+l_{4} Q_{B}^{\prime \prime}\right), \\
{\left[Q_{A}, T^{x \mid a b c}\right]=} & -12 e_{5} \Gamma^{a b c} \sigma_{A}^{x \mid B} Q_{B}^{\prime} \\
{\left[Q_{A}, Z^{a_{1} \ldots a_{5}}\right]=} & -2(5 !) \Gamma^{a_{1} \ldots a_{5}}\left(e_{6} Q_{A}^{\prime}+l_{6} Q_{A}^{\prime \prime}\right), .
\end{aligned}
$$

\subsection{Gauge structure of the minimal $D=7$ FDA}

The gauge structure of the $D=7 \mathrm{FDA}$ can be analyzed in a strictly analogous way as we have done for the $D=11$ case. We limit ourselves to give just a short discussion of it since the relevant point about the role of the nilpotent charges dual to the spinor 1-forms $\eta_{A}$ and $\xi_{A}$ is completely analogous to the one discussed for $\eta$ in the $D=11$ case. The supersymmetric FDA is invariant under the following gauge transformations:

$$
\left\{\begin{array}{l}
\delta A^{x}=d \Lambda^{x} \\
\delta B^{(2)}=d \Lambda^{(1)}-\Lambda^{x} d A^{x} \\
\delta B^{(3)}=d \Lambda^{(2)} \\
\delta A^{x \mid(4)}=d \Lambda^{x \mid(3)}-\frac{1}{2}\left(\Lambda^{x} d B^{(3)}+\Lambda^{(2)} \wedge d A^{x}\right) \\
\delta B^{(6)}=d \Lambda^{(5)}-15 \Lambda^{(2)} \wedge d B^{(3)}
\end{array}\right.
$$

Analogously to the eleven dimensional case, the gauge transformations (4.51) leaving invariant the FDA can be obtained, for particular $(p-1)$-form parameters, through Lie derivatives acting on the hidden symmetry supergroup $G$ underlying the theory. In this case, $G$ has the fiber bundle structure $G=\mathcal{H}+K$, where now $K=G / \mathcal{H}$ is spanned by the supervielbein $\left\{V^{a}, \psi_{A}\right\}$. The fiber $\mathcal{H}=H_{0}+H_{b}+H_{f}$ is generated by the Lorentz generators in $H_{0}$ and by the gauge and hidden generators in $H_{b}$ and $H_{f}$, where now $\left\{T^{x}, Z^{a}, Z^{a b}, T^{x \mid a b c}, Z^{a_{1} \cdots a_{5}}\right\}$ span $H_{b}$, while $\left\{\xi_{A}, \eta_{A}\right\}$ span $H_{f}$.

Explicitly, let us define the tangent vector in $H_{b}$ :

$$
\vec{z} \equiv \Lambda^{x} T^{x}+\Lambda_{a} Z^{a}+\Lambda_{a b} Z^{a b}+\Lambda_{a b c}^{x} T^{x \mid a b c}+\Lambda_{a_{1} \cdots a_{5}} Z^{a_{1} \cdots a_{5}} \in H_{b}
$$


By straightforward calculation we get that the gauge transformations of $A^{x}, B^{(2)}$ and $B^{(3)}$ in (4.51) can be obtained by requiring:

$$
\begin{aligned}
\delta A^{x} & =\ell_{\vec{z}} A^{x}, \\
\delta B^{(2)} & =\ell_{\vec{z}} B^{(2)}, \\
\delta B^{(3)} & =\ell_{\vec{z}} B^{(3)}
\end{aligned}
$$

for the choice of $(p-1)$-form gauge parameters:

$$
\begin{aligned}
\Lambda^{x} & =\imath_{\vec{z}} A^{x}, \\
\Lambda^{(1)} & =\imath_{\vec{z}} B^{(2)}, \\
\Lambda^{(2)} & =\imath_{\vec{z}} B^{(3)},
\end{aligned}
$$

provided the values of the $\tau_{i}$ and $\sigma_{i}$ parameters be given by the equation (B.3) of appendix $\mathrm{B}$, which also assure supersymmetry and consistency of the theory. We expect that in general, also for the forms $A^{x \mid(4)}$ and $B^{(6)}$, whose parametrizations in terms of 1 -forms are still unknown, the rest of the gauge transformations in (4.51) leaving invariant the supersymmetric FDA should be:

$$
\begin{aligned}
\delta A^{x \mid(4)} & =\ell_{\vec{z}} A^{x \mid(4)}, \\
\delta B^{(6)} & =\ell_{\vec{z}} B^{(6)},
\end{aligned}
$$

for the choice of $(p-1)$-form gauge parameters:

$$
\begin{aligned}
\Lambda^{x \mid(3)} & =\imath_{\vec{z}} A^{x \mid(4)}, \\
\Lambda^{(5)} & =\imath_{\vec{z}} B^{(6)} .
\end{aligned}
$$

This corresponds to the following gauge transformations of the 1-forms in $H_{b}$ :

$$
\left\{\begin{array}{l}
\delta A^{x}=d \Lambda^{x}, \\
\delta B_{a}=d \Lambda_{a}, \\
\delta B_{a b}=d \Lambda_{a b}, \\
\delta A_{a b c}^{x}=d \Lambda_{a b c}^{x}, \\
\delta B_{a_{1} \cdots a_{5}}=d \Lambda_{a_{1} \cdots a_{5}},
\end{array}\right.
$$

together with the gauge transformations of the 1-forms in $H_{f}$ :

$$
\left\{\begin{array}{c}
\delta \xi_{A}=D \varepsilon_{A}^{\prime}+e_{2} \Gamma^{a} \psi_{A} \Lambda_{a}+e_{3} \Gamma^{a b} \psi_{A} \Lambda_{a b}+ \\
+e_{4} \psi_{B} \sigma^{x \mid B}{ }_{A} \Lambda^{x}+e_{5} \Gamma^{a b c} \psi_{B} \sigma^{x \mid B}{ }_{A} \Lambda^{x \mid}{ }_{a b c}+e_{6} \Gamma^{a_{1} \ldots a_{5}} \psi_{A} \Lambda_{a_{1} \ldots a_{5}} \\
\delta \eta_{A}=D \varepsilon_{A}^{\prime \prime}+l_{2} \Gamma^{a} \psi_{A} \Lambda_{a}+l_{3} \Gamma^{a b} \psi_{A} \Lambda_{a b}+ \\
+l_{4} \psi_{B} \sigma^{x \mid B}{ }_{A} \Lambda^{x}+l_{5} \Gamma^{a b c} \psi_{B} \sigma^{x \mid B}{ }_{A} \Lambda_{a b c}^{x \mid}+l_{6} \Gamma^{a_{1} \ldots a_{5}} \psi_{A} \Lambda_{a_{1} \ldots a_{5}},
\end{array}\right.
$$

where the parameters $\Lambda_{i \ldots}$ appearing in (4.63) are arbitrary Lorentz (and/or SU(2)) valued 0 -forms while $\varepsilon_{A}^{\prime}, \varepsilon_{A}^{\prime \prime}$ in (4.64) are arbitrary spinor parameters. 


\section{Relation with eleven dimensional supergravity}

The hidden super-Lie algebra discussed in section 4 is the most general one for the $D=7$, $\mathcal{N}=2$ supergravity. Actually, we expect that, for special choices of the parameters, the above structure could be retrieved by dimensional reduction of the eleven dimensional theory, discussed in section 2, in the case where four of the eleven dimensional spacetime directions belong to a four-dimensional compact manifold preserving one-half of the supercharges.

The dimensional reduction of eleven dimensional supergravity to the minimal $D=7$ theory was explicitly performed in [41]. There, it was pointed out that the minimal $D=7$ supergravity theory can be obtained as a truncation of the dimensional reduction of $D=11$ supergravity on a torus $T^{4}$ (that would gives the maximal $D=7$ theory), where the $\mathrm{SO}(4)=\mathrm{SO}(3)_{+} \times \mathrm{SO}(3)_{-}$holonomy on the internal manifold, is truncated to $\mathrm{SO}(3)_{+}$, so that in the truncation only the reduced fields which are $\mathrm{SO}(3)_{-}$-singlets are retained.

As far as the fermionic fields are concerned, the truncation selects only 16 out of the 32 components of the eleven dimensional Majorana spinors, described by pseudo-Majorana spinors valued in the $\mathrm{SU}(2)=\mathrm{SO}(3)_{+}$seven dimensional R-symmetry. In particular, the eleven dimensional gravitino 1-form $\Psi$ becomes, in $D=7$ :

$$
\Psi \rightarrow \psi_{A}, \quad A=1,2 .
$$

As far as the bosonic fields are concerned, let us parametrize the Lie algebra of $\mathrm{SO}(4)$, the holonomy group of the internal manifold, in terms of the four dimensional ' $t$ Hooft matrices $J_{i j}^{x \pm}$, where $x=1,2,3, i, j, \cdots=1, \cdots, 4$ (their properties are recalled in appendix C). The truncation corresponds to drop out the contributions proportional to $J_{i j}^{x-} \in \mathrm{SO}(3)_{-}$ in the decomposition of the eleven dimensional bosonic forms to seven dimensions, so that:

$$
\begin{aligned}
& A^{(3)} \rightarrow B^{(3)}+A^{x} \wedge J_{i j}^{x+} V^{i} \wedge V^{j} \\
& B^{(6)} \rightarrow B^{(6)}+A_{(4)}^{x} \wedge J_{i j}^{x+} V^{i} \wedge V^{j}-8 B^{(2)} \wedge \Omega^{(4)}
\end{aligned}
$$

where $V^{i}$ are the vielbein of the compact manifold and $\Omega^{(4)}=\frac{1}{4 !} V^{i_{1}} \wedge \cdots V^{i_{4}} \epsilon_{i_{1} \cdots i_{4}}$ denotes its volume form.

Next we consider the dimensional reduction of the Lorentz-valued 1-forms $\left\{B_{\hat{a} \hat{b}}, B_{\hat{a}_{1} \cdots \hat{a}_{5}}\right\}$ of eq. (2.19), defining the super-Lie algebra hidden in the FDA in $D=11$, to the minimal $D=7$ theory. We first observe that comparison of the $D=11$ to the $D=7$ theories would generically require to consider the version of the seven dimensional theory which includes the 1-form $B_{a_{1} \cdots a_{5}}$, that in seven dimensions is associated with the (non-dynamical) volume-form $d B^{(6)}$. Indeed by straightforward dimensional reduction we obtain:

$$
\begin{aligned}
& B_{\hat{a} \hat{b}} \rightarrow\left\{\begin{array}{l}
B_{a b} \\
A^{x} J_{i j}^{x+}
\end{array}\right. \\
& B_{\hat{a}_{1} \cdots \hat{a}_{5}} \rightarrow\left\{\begin{array}{l}
B_{a_{1} \cdots a_{5}} \\
-\frac{3 \mathrm{i}}{2} A_{a b c}^{x} J_{i j}^{x+} \\
-B_{a} \epsilon_{i_{1} \cdots i_{4}}
\end{array}\right.
\end{aligned}
$$


where $\hat{a}=0,1, \cdots 10 ; a=0,1, \cdots 6 ; i=7, \cdots 10$. Note that to neglect $B_{\hat{a}_{1} \cdots \hat{a}_{5}}$ would imply, for consistency of the dimensional reduction, to drop out also all the other forms in (5.5).

As it was observed previously, the hidden superalgebra in $D=11$ was obtained in [6] by parametrizing only the 3 -form $A^{(3)}$ in terms of 1 -forms, while the parametrization of the Hodge-dual potential $B^{(6)}$ was not considered there. For this reason we are going to compare the dimensional reduction of $D=11$ fields considering only the fields appearing in the parametrization of the 3 -form. In light of the fact that the $D=7$ field $B^{(2)}$ descends from the $D=116$-form $B^{(6)}$ (see eq. (5.3)), comparison of the two theories could shed some light on the parametrization of the $D=11$ form $B^{(6)}$ and then in the full hidden superalgebra of the $D=11$ theory, since we cannot get any direct information on the parametrization of $B^{(6)}$ from the results of [6] reviewed in section 2. In particular, the analysis given in section 4 shows that the full hidden super algebra in $D=7$ also includes a second nilpotent spin-3/2 field appearing in the parametrization of $B^{(2)}$, see eq. (4.13). As $B^{(2)}$ is a descendent of $B^{(6)}$ from eleven to seven dimensions, this could suggest that considering also the parametrization of $B^{(6)}$ in the analysis of the $D=11$ hidden structure, would amount to include one extra nilpotent fermionic 1-form $\eta^{\prime}$. A verification of this conjecture by an explicit calculation is left to a future investigation.

Let us quote the set of relations that we found between the $D=7$ and $D=11$ structure constants:

$$
\begin{array}{rrrl}
e_{1} & =\mathrm{i} E_{1}, & e_{2}=-360 E_{3}, & e_{3}=E_{2}, \\
e_{4}=4 \mathrm{i} E_{2}, & e_{5}=120 E_{3}, & e_{6}=\mathrm{i} E_{3} .
\end{array}
$$

The corresponding relations between the coefficients in the parametrizations of the 3form are:

$$
\begin{aligned}
& \tau_{0}=1, \quad \tau_{1}=0, \\
& \tau_{4}=7200 T_{3}, \quad \tau_{5}=-1296 T_{4}, \\
& \tau_{8}=-4 T_{1}, \quad \tau_{9}=216 \times 180 T_{4}, \\
& \tau_{12}=-240 T_{3}, \quad \tau_{13}=0, \\
& \begin{aligned}
\tau_{2} & =-\frac{3}{8} T_{2}, & \tau_{3} & =\frac{1}{2} T_{1}, \\
\tau_{6} & =-216 T_{2}, & \tau_{7} & =144 T_{2}, \\
\tau_{10} & =T_{2}, & \tau_{11} & =0,
\end{aligned} \\
& \tau_{14}=1944 T_{4} \text {. }
\end{aligned}
$$

In particular, we note that in the dimensional reduced theory $\tau_{1}=0, \tau_{11}=0$, and $\tau_{13}=0$.

Curiously enough, requiring that the set of coefficients (5.6) and (5.7) satisfy the general relations (4.17) of the seven dimensional theory, implies the condition $T_{0}=1$ on the set of coefficients of the $D=11$ theory, thus selecting the particular solution (A.3) originally found in [6].

We finally write down the hidden superalgebra in the $D=7$ theory obtained by dimensional reduction from $D=11$ :

$$
\begin{aligned}
\left\{Q^{A}, \bar{Q}_{B}\right\} & =-\mathrm{i} \Gamma^{a}\left(P_{a}+\eta_{a b} Z^{b}\right) \delta_{B}^{A}-\frac{1}{2} \Gamma_{a b} Z_{B}^{a b} \delta_{B}^{A}-\sigma_{B}^{x \mid A}\left(\mathrm{i} T^{x}+\frac{1}{18} \Gamma_{a b c} T^{x \mid a b c}\right) \\
{\left[Q_{A}, P_{a}\right] } & =-2 \mathrm{i}\left(\begin{array}{c}
5 E_{2} \\
0
\end{array}\right) \Gamma_{a} Q_{A}^{\prime}
\end{aligned}
$$




$$
\begin{aligned}
{\left[Q_{A}, Z^{a}\right] } & =-720\left(\begin{array}{c}
E_{2} / 48 \\
E_{2} / 72
\end{array}\right) \Gamma^{a} Q_{A}^{\prime}, \\
{\left[Q_{A}, Z^{a b}\right] } & =-4 E_{2} \Gamma^{a b} Q_{A}^{\prime}, \\
{\left[Q_{A}, T^{x}\right] } & =-8 \mathrm{i} E_{2} \sigma^{x \mid B}{ }_{A} Q_{B}^{\prime}, \\
{\left[Q_{A}, T^{x \mid a b c}\right] } & =-1440\left(\begin{array}{c}
E_{2} / 48 \\
E_{2} / 72
\end{array}\right) \Gamma^{a b c} \sigma_{A}^{x \mid B} Q_{B}^{\prime}, \\
{\left[Q_{A}, Z^{a_{1} \ldots a_{5}}\right] } & =-2(5 !) \mathrm{i}\left(\begin{array}{c}
E_{2} / 48 \\
E_{2} / 72
\end{array}\right) \Gamma^{a_{1} \ldots a_{5}} Q_{A}^{\prime} .
\end{aligned}
$$

We see that there are indeed two inequivalent solutions, distinguished by the set of structure constants involving $Q_{A}^{\prime}$. In particular the second one features the peculiarity that the commutator $\left[Q_{A}, P_{a}\right]$ vanishes in corerespondence of the solution $e_{1}=E_{1}=0$. We see that this second solution has a special meaning in the $D=7$ theory: it can be obtained as a special case if we further require the following identification to hold in the seven dimensional theory:

$$
B^{a_{1} \ldots a_{5}}=\frac{1}{2} B_{a b} \epsilon^{a_{1} \ldots a_{5} a b} .
$$

The identification is possible in $D=7$ due to the actual degeneration of the Lorentz-index structure for the two 1-forms in (5.15). However, in the parent $D=11$ theory the two 1forms that get identified through (5.15) are associated with the mutually non-local exterior forms $A^{(3)}$ and $B^{(6)}$. We speculate that the absence of the coupling of the translation generator to $Q^{\prime}$ in this case could possibly be related to the intrinsically topological $D=11$ structure inherent in the association (5.15).

\section{Concluding remarks}

In this paper we have reconsidered the hidden superalgebra structure that underlies supergravity theories in space-time dimensions $D>5$ (and in general the supersymmetric theories involving $p$-form gauge fields with $p>1$ ), first introduced in [6] in the $\mathrm{D}=11$ theory. It generalizes the supersymmetry algebra to include the set of almost-central charges (carrying Lorentz indices) which are currently associated with $(p-1)$-brane charges. We focussed in particular on the role played by the nilpotent spinor charges naturally appearing in the hidden superalgebra when constructed in the geometrical approach, showing that such extra charges, besides allowing the closure of the algebra, are also necessary in order for the FDA to be supersymmetric and gauge invariant on superspace.

Considering in detail the $\mathrm{D}=11$ case, we clarified the physical interpretation of the spinor 1-form field dual to the nilpotent spinor charge: it is not a physical field in superspace, its differential being parametrized in an enlarged superspace which includes the almost-central charges as bosonic tangent space generators, besides the supervielbein $\left\{V^{a}, \psi^{\alpha}\right\}$. Precisely because of this feature, it guarantees that instead the 1-forms dual to the almost central charges are genuine abelian gauge fields whose generators, together with the nilpotent fermionic generators, close an abelian ideal of the supergroup. 
As the generators of the hidden super Lie algebra span the tangent space of a supergroup manifold, then in our geometrical approach the fields are naturally defined in an enlarged manifold corresponding to the supergroup manifold, where all the invariances of the FDA are diffeomorphisms, generated by Lie derivatives. The spinor 1-form allows, in a dynamical way, the diffeomorphisms in the directions spanned by the almost central charges to be particular gauge transformations, so that one obtains the ordinary superspace as the quotient of the supergroup over the fiber subgroup of gauge transformations.

We have further considered a lower dimensional case, with the aim to investigate a possible enlargement of the hidden supergroup structure found in $D=11$, focussing in particular on the minimal $\mathrm{D}=7 \mathrm{FDA}$. Indeed, in that case we were able to parametrize in terms of 1-forms the couple of mutually non-local forms $B^{(2)}$ and $B^{(3)}$. An analogous investigation in $\mathrm{D}=11$ would have required the knowledge of the explicit parametrization of $B^{(6)}$, which is mutually non-local with $A^{(3)}$, but which at the moment has not yet been worked out. In the seven dimensional case we found that two nilpotent spinor 1forms are required to find the most general hidden Lie superalgebra. However, as was to be expected, in this case we found that two subalgebras exist, where only one spinor, parametrizing only one of the two mutually non-local $p$-forms, is present. We called them Lagrangian subalgebras, since they should correspond to the expected symmetries of a lagrangian description of the theory in terms of 1-forms, or, for the corresponding FDA, to the presence of either $B^{(2)}$ or $B^{(3)}$ in the lagrangian.

The above results point out to the possible existence of an enlargement also of the $\mathrm{D}=11$ hidden superalgebra, associated with further spinor 1-forms in the parametrization of $B^{(6)}$. This possibility is currently under investigation.

Our results could be extended in several directions which are left to future investigation.

A relevant issue is the analysis of the hidden structure once gauge charges are included in the FDA. Moreover, it would be interesting to consider the dynamical theory based on the 1-form formulation of the supersymmetric FDA, including coupling to matter and more generally a gauging of the theory. A lagrangian description of the interacting theory should be based on one of the Lagrangian subalgebras of the relevant hidden supergroup. Finally, we observe that the framework worked out in this paper is naturally related to the formulation of double field theory and its generalizations. As we have seen, the consistency of our framework is implemented dynamically by the very presence of the nilpotent spinor generators in the hidden subalgebra, so that we are led to conjecture that the consistency constraints required in double field theory could be proficiently expressed in our geometrical framework.

\section{Acknowledgments}

We have benefited of stimulating discussions with our friend Mario Trigiante and we thank him for a critical reading of the manuscript. 


\section{A The explicit solution for $A^{(3)}$ in $D=11$}

In $D=11$, requiring consistency of the parametrization of the 3 -form $A^{(3)}$, see equation (2.9), the following set of equations must be satisfied

$$
\left\{\begin{array}{l}
T_{0}-2 S_{1} E_{1}-1=0 \\
T_{0}-2 S_{1} E_{2}-2 S_{2} E_{1}=0 \\
3 T_{1}-8 S_{2} E_{2}=0 \\
T_{2}+10 S_{2} E_{3}+10 S_{3} E_{2}=0 \\
120 T_{3}-S_{3} E_{1}-S_{1} E_{3}=0 \\
T_{2}+1200 S_{3} E_{3}=0 \\
T_{3}-2 S_{3} E_{3}=0 \\
9 T_{4}+10 S_{3} E_{3}=0 \\
S_{1}+10 S_{2}-720 S_{3}=0
\end{array}\right.
$$

while the integrability condition $D^{2} \eta=0$ further implies:

$$
E_{1}+10 E_{2}-720 E_{3}=0 .
$$

Here we have also correct some misprints appearing in $[6]$ and $[16,17]$. This system is solved by the relations (2.11).

In [6] the first coefficient $T_{0}$ was arbitrarily fixed to $T_{0}=1$; if we then fix the normalization $T_{0}=1$ in our system, we get two distinct solutions, depending on the parameter $E_{2}$ (which just fixes the normalization of $\eta$ ):

$$
\begin{aligned}
& T_{0}=1, \quad T_{1}=\frac{4}{15}, \quad T_{2}=-\frac{5}{144}, \quad T_{3}=\frac{1}{17280}, \quad T_{4}=-\frac{1}{31104}, \\
& S_{1}=\left(\begin{array}{c}
0 \\
\frac{1}{2 E_{2}}
\end{array}\right), \quad S_{2}=\frac{1}{10 E_{2}}, \quad S_{3}=\left(\begin{array}{c}
\frac{1}{720 E_{2}} \\
\frac{1}{480 E_{2}}
\end{array}\right), \quad E_{1}=\left(\begin{array}{c}
5 E_{2} \\
0
\end{array}\right), \quad E_{3}=\left(\begin{array}{c}
\frac{E_{2}}{48} \\
\frac{E_{2}}{72}
\end{array}\right) \text {. }
\end{aligned}
$$

\section{B The explicit solution for $B^{(2)}$ and $B^{(3)}$ in $D=7$}

As far as the parametrization of $B^{(2)}$ and $B^{(3)}$ are concerned we distinguish between the case with $B_{a_{1} \cdots a_{5}}=0$ and $B_{a_{1} \cdots a_{5}} \neq 0$.

\section{B.1 Coefficients in the case $B_{a_{1} \cdots a_{5}}=0$}

- Coefficients in the parametrization of $B^{(2)}$

The coefficients are given by:

$$
\sigma=2 \mathrm{i} l_{2} \tau, \quad l_{1}=\frac{\mathrm{i}}{2 \tau}\left(-1+2 \mathrm{i} l_{2} \tau\right), \quad l_{3}=0, \quad l_{4}=\frac{\mathrm{i}}{2 \tau}, \quad l_{5}=0, .
$$

- Coefficients in the parametrization of $B^{(3)}$ 
If we factorize

$$
\frac{e_{5}}{\sigma_{5}}=H \equiv-2\left[e_{1}-2 \mathrm{i}\left(e_{3}+\frac{\mathrm{i}}{2} e_{2}\right)\right]\left[e_{1}-2 \mathrm{i}\left(\frac{\mathrm{i}}{2} e_{2}+e_{5}\right)\right],
$$

we can write the coefficients in the following form:

$$
\begin{aligned}
& \tau_{0}=8\left[\frac{\mathrm{i}}{2} e_{1}\left(e_{3}-e_{5}\right)+\left(\frac{\mathrm{i}}{2} e_{2}+e_{3}\right)\left(\frac{\mathrm{i}}{3} e_{2}+e_{5}\right)\right] \frac{\sigma_{5}}{e_{5}} \\
& \tau_{1}=-8 \mathrm{i} e_{2}\left(\frac{\mathrm{i}}{2} e_{2}+e_{5}\right) \frac{\sigma_{5}}{e_{5}}, \quad \tau_{2}=-2 e_{2}^{2} \frac{\sigma_{5}}{e_{5}}, \quad \tau_{3}=-\frac{16 e_{3}\left(\frac{1}{2} e_{3}-e_{5}\right)}{3} \frac{\sigma_{5}}{e_{5}} \\
& \tau_{4}=2\left(\frac{\mathrm{i}}{2} e_{2}+e_{5}\right) \sigma_{5}, \quad \tau_{5}=-\mathrm{i} e_{2} \sigma_{5}, \quad \tau_{6}=36 e_{5} \sigma_{5} \\
& \tau_{7}=-12 e_{5} \sigma_{5}, \quad \tau_{8}=\frac{2}{3} e_{4}\left[e_{1}-2 \mathrm{i}\left(-3 e_{3}+\frac{\mathrm{i}}{2} e_{2}+3 e_{5}\right)\right] \frac{\sigma_{5}}{e_{5}}, \quad \tau_{9}=-3 e_{5} \sigma_{5} \\
& \sigma_{1}=\left[-e_{1}+4 \mathrm{i}\left(\frac{\mathrm{i}}{2} e_{2}+e_{5}\right)\right] \frac{\sigma_{5}}{e_{5}}, \quad \sigma_{2}=e_{2} \frac{\sigma_{5}}{e_{5}}, \\
& \sigma_{3}=-2\left(\frac{1}{2} e_{3}-e_{5}\right) \frac{\sigma_{5}}{e_{5}}, \quad \sigma_{4}=-e_{4} \frac{\sigma_{5}}{e_{5}}, \quad \sigma_{5}=\frac{e_{5}}{H} \\
& e_{4}=-e_{1}+2 \mathrm{i}\left(-3 e_{3}+\frac{\mathrm{i}}{2} e_{2}+5 e_{5}\right) .
\end{aligned}
$$

In the relations above, the set of coefficients $\left\{\sigma_{i}\right\}$, that multiply the fermion bilinears in the parametrization of $B^{(3)}$, are given in terms of the structure constants $\left\{e_{i}\right\}$ appearing in $D \xi_{A}$. It is noteworthy that the inverse transformation expressing the $\left\{e_{i}\right\}$ in terms of the $\left\{\sigma_{i}\right\}$ has exactly the same form, since the system of equations is completely symmetric in the interchange of them.

\section{B.2 Coefficients in the case $B_{a_{1} \cdots a_{5}} \neq 0$}

- Coefficients in the parametrization of $B^{(2)}$

$$
\begin{array}{lll}
\sigma=2 \mathrm{i} l_{2} \tau, & l_{1}=\frac{\mathrm{i}}{2 \tau}\left(-1+2 \mathrm{i} l_{2} \tau\right), & l_{3}=-\frac{60 \chi}{\tau}, \\
l_{4}=\frac{\mathrm{i}}{2 \tau}, & l_{5}=0, & l_{6}=\mathrm{i} \frac{\chi}{\tau} .
\end{array}
$$

- Coefficients in the parametrization of $B^{(3)}$

If we factorize

$$
\frac{e_{5}}{\sigma_{5}}=\hat{H} \equiv-2\left[e_{1}-2 \mathrm{i}\left(e_{3}+\frac{\mathrm{i}}{2} e_{2}-60 \mathrm{i} e_{6}\right)\right]\left[e_{1}-2 \mathrm{i}\left(\frac{\mathrm{i}}{2} e_{2}+e_{5}\right)\right]
$$

we can write the coefficients in the following form:

$$
\begin{aligned}
\tau_{0} & =\frac{2 e_{1}\left[e_{1}-4 \mathrm{i}\left(\frac{\mathrm{i}}{2} e_{2}+e_{5}\right)\right]}{\hat{H}}+120 \tau_{11}+1, \\
\tau_{1} & =-4\left[\frac{4\left(\frac{\mathrm{i}}{2} e_{2}+e_{5}\right)\left(\frac{\mathrm{i}}{2} e_{2}-60 \mathrm{i} e_{6}\right)}{\hat{H}}+60 \tau_{11}+\frac{360 e_{1} e_{6}\left(\frac{1}{6} e_{5}+20 \mathrm{i} e_{6}\right)}{\hat{H}\left(\frac{1}{2} e_{3}+90 \mathrm{i} e_{6}\right)}\right],
\end{aligned}
$$




$$
\begin{aligned}
& \tau_{2}=120\left[\tau_{11}-\frac{\mathrm{i} e_{6}\left(\mathrm{i} e_{1}+2 \mathrm{i} e_{2}+4 e_{5}\right)-\mathrm{i} e_{2}\left(\frac{\mathrm{i}}{2} e_{2}-30 \mathrm{i} e_{6}\right)}{\hat{H}}\right] \\
& +\frac{120\left(e_{1}-e_{2}\right) e_{6}\left(-\frac{1}{2} e_{3}+e_{5}+30 \mathrm{i} e_{6}\right)}{\hat{H}\left(\frac{1}{2} e_{3}+90 \mathrm{i} e_{6}\right)}, \\
& \tau_{3}=-\frac{16 e_{3}\left(\frac{1}{2} e_{3}-e_{5}-30 \mathrm{i} e_{6}\right)\left(\frac{1}{2} e_{3}+60 \mathrm{i} e_{6}\right)}{3 \hat{H}\left(\frac{1}{2} e_{3}+90 \mathrm{i} e_{6}\right)}, \quad \tau_{4}=\frac{2 e_{5}\left(\frac{\mathrm{i}}{2} e_{2}+e_{5}\right)}{\hat{H}}, \quad \tau_{5}=-\frac{\mathrm{i} e_{2} e_{5}}{\hat{H}}, \\
& \tau_{6}=\frac{216 e_{5}\left[60 \mathrm{i} e_{6}\left(\frac{1}{6} e_{5}+5 \mathrm{ie}_{6}\right)+\frac{1}{2} e_{3}\left(\frac{1}{6} e_{5}+10 \mathrm{i} e_{6}\right)\right]}{\hat{H}\left(\frac{1}{2} e_{3}+60 \mathrm{i} e_{6}\right)}, \quad \tau_{7}=-\frac{12 e_{5}^{2}}{\hat{H}}, \\
& \tau_{8}=-\frac{2}{3} \frac{\left[e_{1}-2 \mathrm{i}\left(\frac{\mathrm{i}}{2} e_{2}-3 e_{3}+3 e_{5}+180 \mathrm{i} e_{6}\right)\right]\left[e_{1}-2 \mathrm{i}\left(\frac{\mathrm{i}}{2} e_{2}-3 e_{3}+5 e_{5}+180 \mathrm{i} e_{6}\right)\right]}{\hat{H}}, \\
& \tau_{9}=-\frac{3 e_{5}^{2}}{\hat{H}}, \quad \tau_{10}=-\frac{1200 e_{6}^{2}\left(-\frac{1}{2} e_{3}+e_{5}+30 \mathrm{i} e_{6}\right)}{\hat{H}\left(\frac{1}{2} e_{3}+90 \mathrm{i} e_{6}\right)}, \\
& \tau_{11}=\tau_{11}, \quad \tau_{12}=\frac{4 e_{6}\left[3 \mathrm{i} e_{1}\left(\frac{1}{6} e_{5}+20 \mathrm{i} e_{6}\right)+2\left(\frac{\mathrm{i}}{2} e_{2}+e_{5}\right)\left(\frac{1}{2} e_{3}+90 \mathrm{i} e_{6}\right)\right]}{\hat{H}\left(\frac{1}{2} e_{3}+90 \mathrm{i} e_{6}\right)}-2 \tau_{11}, \\
& \tau_{13}=\frac{-2 \mathrm{i} e_{6}\left[\frac{\mathrm{i}}{2}\left(e_{1}-e_{2}\right) e_{5}+2 e_{3}\left(\frac{\mathrm{i}}{2} e_{2}+\frac{1}{2} e_{5}\right)+60 \mathrm{i} e_{6}\left(\mathrm{i} e_{1}+2 \mathrm{i} e_{2}+3 e_{5}\right)\right]}{\hat{H}\left(\frac{1}{2} e_{3}+90 \mathrm{i} e_{6}\right)}+\tau_{11}, \\
& \tau_{14}=\frac{18 \mathrm{i} e_{5} e_{6}\left(\frac{1}{2} e_{3}-\frac{1}{2} e_{5}+30 \mathrm{i} e_{6}\right)}{\hat{H}\left(\frac{1}{2} e_{3}+90 \mathrm{i} e_{6}\right)} \\
& \sigma_{1}=-\frac{e_{1}-4 \mathrm{i}\left(\frac{\mathrm{i}}{2} e_{2}+e_{5}\right)}{\hat{H}}, \quad \sigma_{2}=\frac{e_{2}}{\hat{H}}, \quad \sigma_{3}=-\frac{2\left(\frac{1}{2} e_{3}-e_{5}-30 \mathrm{i} e_{6}\right)\left(\frac{1}{2} e_{3}+60 \mathrm{i} e_{6}\right)}{\hat{H}\left(\frac{1}{2} e_{3}+90 \mathrm{i} e_{6}\right)}, \\
& \sigma_{4}=\frac{e_{1}-2 \mathrm{i}\left(\frac{\mathrm{i}}{2} e_{2}-3 e_{3}+3 e_{5}+180 \mathrm{i} e_{6}\right)}{\hat{H}}, \quad \sigma_{5}=\frac{e_{5}}{\hat{H}}, \quad \sigma_{6}=\frac{e_{6}\left(\frac{1}{2} e_{3}-e_{5}-30 \mathrm{i} e_{6}\right)}{\hat{H}\left(\frac{1}{2} e_{3}+90 \mathrm{i} e_{6}\right)}, \\
& e_{4}=-e_{1}+2 \mathrm{i}\left(-3 e_{3}+\frac{\mathrm{i}}{2} e_{2}+5 e_{5}+180 \mathrm{i} e_{6}\right) \text {. }
\end{aligned}
$$

\section{Dimensional reduction of the gamma matrices}

In this section we write the dimensional reduction of the gamma matrices from $D=11$ to $D=7$ dimensions. We decompose the gamma matrices in eleven dimensions (hatted ones) in the following way:

$$
\hat{\Gamma}_{\hat{a}} \rightarrow \begin{cases}4 D & \Gamma_{i}, \\ 7 D & \Gamma_{a},\end{cases}
$$

where $\hat{a}=0, \ldots, 10, a=0, \ldots, 6$, and $i=7,8,9,10$. Then we can write the following decomposition:

$$
\begin{aligned}
\Gamma_{i} & =\mathbb{1}_{4} \otimes \gamma_{i}, \\
\Gamma_{a} & =\Gamma_{a} \otimes \gamma_{5},
\end{aligned}
$$

where

$$
\gamma_{5}=\left(\begin{array}{cc}
\delta_{A}^{B} & 0 \\
0 & -\delta_{A^{\prime}}^{B^{\prime}}
\end{array}\right), \quad \gamma^{5}=\mathbb{1}_{4}
$$


and

$$
\gamma_{i}=\left(\begin{array}{cc}
0 & \left(\gamma_{i}\right){ }_{A}^{A^{\prime}} \\
\left(\gamma_{i}\right)_{A^{\prime}}^{A} & 0
\end{array}\right), \quad\left\{\gamma_{i}, \gamma_{j}\right\}=2 \eta_{i j}=-2 \delta_{i j},
$$

where $i, j, \ldots$ are the internal index running from 7 to 10 and we are using a mostly minus Minkowski metric. Thus we can write:

$$
\begin{aligned}
\Gamma_{a} & =\left(\begin{array}{cc}
\left(\Gamma_{a}\right)_{\alpha}^{\beta} \delta_{A}^{B} & \mathbf{0} \\
\mathbf{0} & -\left(\Gamma_{a}\right)_{\alpha}^{\beta} \delta_{A^{\prime}}^{B^{\prime}}
\end{array}\right), \\
\Gamma_{i} & =\left(\begin{array}{cc}
\mathbf{0} & \left(\gamma_{i}\right)_{A}^{A^{\prime}} \delta_{\alpha}^{\beta} \\
\left(\gamma_{i}\right)_{A^{\prime}}^{A} \delta_{\alpha}^{\beta} & \mathbf{0}
\end{array}\right) .
\end{aligned}
$$

\section{C.1 Properties of the 't Hooft matrices}

The self-dual and antiself-dual 't Hooft matrices satisfy the quaternionic algebra:

$$
\begin{aligned}
J^{ \pm \mid x} J^{ \pm \mid y} & =-\delta^{x y} \mathbb{1}_{4 \times 4}+\epsilon^{x y z} J^{ \pm \mid z}, \\
J_{a b}^{ \pm \mid x} & = \pm \frac{1}{2} \epsilon_{a b c d} J_{c d}^{ \pm \mid x} \\
{\left[J^{+\mid x}, J^{-\mid y}\right] } & =0, \quad \forall x, y,
\end{aligned}
$$

from which it follows

$$
\operatorname{Tr}\left(J_{r s}^{x} J_{s t}^{y} J_{t r}^{z}\right)=\operatorname{Tr}\left(\epsilon^{x y z^{\prime}} J^{z^{\prime}} J^{z}\right)=\operatorname{Tr}\left(-\epsilon^{x y z^{\prime}} \delta^{z z^{\prime}} \mathbb{1}_{4}\right)=-4 \epsilon^{x y z} .
$$

\section{Fierz identities and irreducible representations}

\section{D.1 3-gravitino irreducible representations in $D=11$}

The gravitino 1 -form $\Psi_{\alpha},(\alpha=1, \cdots, 32)$, of eleven dimensional supergravity is a commuting spinor 1-form belonging to the spinor representation of $\mathrm{SO}(1,10) \simeq \operatorname{Spin}(32)$. The symmetric product $(\alpha, \beta, \gamma) \equiv \Psi_{(\alpha} \wedge \Psi_{\beta} \wedge \Psi_{\gamma)}$, whose dimension is $\mathbf{5 9 8 4}$, belongs to the three-times symmetric reducible representation of $\operatorname{Spin}(32)$ : The Fierz identities amount to decompose the given representation $(\alpha, \beta, \gamma)$ into irrepses of $\operatorname{Spin}(32)$. One obtains:

$$
5984 \rightarrow 32+320+1408+4224
$$

and the corresponding irreducible spinor representations of the Lorentz group $\mathrm{SO}(1,10)$ will be denoted as follows:

$$
\Xi^{(32)} \in \mathbf{3 2}, \quad \Xi_{a}^{(320)} \in \mathbf{3 2 0}, \quad \Xi_{a_{1} a_{2}}^{(1408)} \in \mathbf{1 4 0 8}, \quad \Xi_{a_{1} \ldots a_{5}}^{(4224)} \in \mathbf{4 2 2 4},
$$

where the indices $a_{1} \cdots a_{n}$ are antisymmetrized, and each of them satisfies $\Gamma^{a} \Xi_{a b_{1} \cdots b_{n}}=0$. One can easily compute the coefficients of the explicit decomposition into the irreducible basis, obtaining: [6, 37]:

$$
\begin{aligned}
& \Psi \wedge \bar{\Psi} \wedge \Gamma_{a} \Psi=\Xi_{a}^{(320)}+\frac{1}{11} \Gamma_{a} \Xi^{(32)}, \\
& \Psi \wedge \bar{\Psi} \Gamma_{a_{1} a_{2}} \Psi=\Xi_{a_{1} a_{2}}^{(1408)}-\frac{2}{9} \Gamma_{\left[a_{2}\right.} \Xi_{\left.a_{2}\right]}^{(320)}+\frac{1}{11} \Gamma_{a_{1} a_{2}} \Xi^{(32)}, \\
& \Psi \wedge \bar{\Psi} \wedge \Gamma_{a_{1} \ldots a_{5}} \Psi=\Xi_{a_{1} \ldots a_{5}}^{(4224)}+2 \Gamma_{\left[a_{1} a_{2} a_{3}\right.} \Xi_{\left.a_{4} a_{5}\right]}^{(1408)}+\frac{5}{9} \Gamma_{\left[a_{1} \ldots a_{4}\right.} \Xi_{\left.a_{5}\right]}^{(320)}-\frac{1}{77} \Gamma_{a_{1} \ldots a_{5}} \Xi^{(32)} \text {. }
\end{aligned}
$$




\section{D.2 Irreducible representations in $D=7$}

An analogous decomposition in seven dimensions gives:

$$
\begin{aligned}
& \psi_{C} \wedge \bar{\psi}^{C} \wedge \psi_{A}=\Xi_{A} \\
& \psi_{A} \wedge \bar{\psi}^{C} \wedge \Gamma^{a b} \psi_{C}=\Xi_{A}^{a b}-\frac{2}{5} \Gamma^{[a} \Xi_{A}^{b]}+\frac{2}{7} \Gamma^{a b} \Xi_{A}, \\
& \psi_{A} \wedge \bar{\psi}^{C} \wedge \Gamma^{a} \psi_{C}=\Xi_{A}^{a}+\frac{2}{7} \Gamma^{a} \Xi_{A}, \\
& \psi_{(A} \wedge \bar{\psi}_{B} \wedge \psi_{C)}=\Xi_{(A B C)} \\
& \psi_{C} \wedge \bar{\psi}^{C} \wedge \Gamma^{a b c} \psi_{A}= \frac{3}{2} \Gamma^{[a} \Xi_{A}^{b c]}+\frac{9}{10} \Gamma^{[a b} \Xi_{A}^{c]}-\frac{1}{7} \Gamma^{a b c} \Xi_{A}, \\
& \psi^{C} \wedge \bar{\psi}^{A} \wedge \Gamma^{a b c} \psi^{B}= \Xi^{(A B C) \mid a b c}+\frac{1}{5} \Gamma^{a b c} \Xi^{(A B C)}+ \\
&\left.-\frac{2}{3} \epsilon^{C(A}\left(\frac{3}{2} \Gamma^{[a} \Xi^{b c] \mid B)}+\frac{9}{10} \Gamma^{[a b} \Xi^{c] \mid B)}-\frac{1}{7} \Gamma^{a b c} \Xi^{\mid} B\right)\right), \\
& \psi^{C} \wedge \bar{\psi}^{A} \wedge \psi^{B}= \Xi^{(A B C)}-\frac{2}{3} \epsilon^{C(A} \Xi^{B)} .
\end{aligned}
$$

Open Access. This article is distributed under the terms of the Creative Commons Attribution License (CC-BY 4.0), which permits any use, distribution and reproduction in any medium, provided the original author(s) and source are credited.

\section{References}

[1] M.B. Green, J.H. Schwarz and E. Witten, Superstring theory. Vol. 1: introduction, Cambridge Monographs On Mathematical Physics, Cambridge University Press, Cambridge U.K. (1987).

[2] M.B. Green, J.H. Schwarz and E. Witten, Superstring theory. Vol. 2: loop amplitudes, anomalies and phenomenology, Cambridge Monographs On Mathematical Physics, Cambridge University Press, Cambridge U.K. (1987).

[3] J. Polchinski, String theory. Vol. 1: an introduction to the bosonic string, Cambridge Monographs On Mathematical Physics, Cambridge University Press, Cambridge U.K. (2005).

[4] J. Polchinski, String theory. Vol. 2: Superstring theory and beyond, Cambridge Monographs On Mathematical Physics, Cambridge University Press, Cambridge U.K. (2005).

[5] E. Cremmer, B. Julia and J. Scherk, Supergravity theory in eleven-dimensions, Phys. Lett. B 76 (1978) 409 [INSPIRE].

[6] R. D'Auria and P. Fré, Geometric supergravity in D=11 and its hidden supergroup, Nucl. Phys. B 201 (1982) 101 [Erratum ibid. B 206 (1982) 496] [INSPIRE].

[7] R. Haag, J.T. Lopuszanski and M. Sohnius, All possible generators of supersymmetries of the s matrix, Nucl. Phys. B 88 (1975) 257 [INSPIRE].

[8] E. Witten and D.I. Olive, Supersymmetry algebras that include topological charges, Phys. Lett. B 78 (1978) 97 [INSPIRE]. 
[9] J.W. van Holten and A. Van Proeyen, $N=1$ supersymmetry algebras in $D=2, D=3$, $D=4$ MOD-8, J. Phys. A 15 (1982) 3763 [inSPIRE].

[10] A. Achucarro, J.M. Evans, P.K. Townsend and D.L. Wiltshire, Super p-branes, Phys. Lett. B 198 (1987) 441 [INSPIRE].

[11] J.A. de Azcarraga, J.P. Gauntlett, J.M. Izquierdo and P.K. Townsend, Topological extensions of the supersymmetry algebra for extended objects, Phys. Rev. Lett. 63 (1989) 2443 [INSPIRE].

[12] E.R.C. Abraham and P.K. Townsend, Intersecting extended objects in supersymmetric field theories, Nucl. Phys. B 351 (1991) 313 [inSPIRE].

[13] J. Polchinski, Dirichlet branes and Ramond-Ramond charges, Phys. Rev. Lett. 75 (1995) 4724 [hep-th/9510017] [INSPIRE].

[14] C.M. Hull and P.K. Townsend, Unity of superstring dualities, Nucl. Phys. B 438 (1995) 109 [hep-th/9410167] [INSPIRE].

[15] P.K. Townsend, P-brane democracy, in The world in eleven dimensions, M.J. Duff ed., CRC Press, U.S.A. (1999), hep-th/9507048 [INSPIRE].

[16] I.A. Bandos, J.A. de Azcarraga, J.M. Izquierdo, M. Picón and O. Varela, On the underlying gauge group structure of D=11 supergravity, Phys. Lett. B 596 (2004) 145 [hep-th/0406020] [INSPIRE].

[17] I.A. Bandos, J.A. de Azcarraga, J.M. Izquierdo, M. Picón and O. Varela, On the underlying gauge group structure of $D=11$ supergravity, Phys. Lett. B 596 (2004) 145 [hep-th/0406020] [INSPIRE].

[18] N. Hitchin, Generalized Calabi-Yau manifolds, Quart. J. Math. 54 (2003) 281 [math/0209099] [INSPIRE].

[19] M. Gualtieri, Generalized complex geometry, math/0703298 [INSPIRE].

[20] M. Graña, Flux compactifications in string theory: a comprehensive review, Phys. Rept. 423 (2006) 91 [hep-th/0509003] [INSPIRE].

[21] C.M. Hull and R.A. Reid-Edwards, Flux compactifications of string theory on twisted tori, Fortsch. Phys. 57 (2009) 862 [hep-th/0503114] [INSPIRE].

[22] A. Dabholkar and C. Hull, Generalised T-duality and non-geometric backgrounds, JHEP 05 (2006) 009 [hep-th/0512005] [INSPIRE].

[23] M. Graña, R. Minasian, M. Petrini and D. Waldram, T-duality, generalized geometry and non-geometric backgrounds, JHEP 04 (2009) 075 [arXiv:0807.4527] [INSPIRE].

[24] C.M. Hull, Generalised Geometry for M-theory, JHEP 07 (2007) 079 [hep-th/0701203] [INSPIRE].

[25] P. Pires Pacheco and D. Waldram, M-theory, exceptional generalised geometry and superpotentials, JHEP 09 (2008) 123 [arXiv:0804.1362] [INSPIRE].

[26] A. Coimbra, C. Strickland-Constable and D. Waldram, Supergravity as generalised geometry II: $E_{d(d)} \times \mathbb{R}^{+}$and M-theory, JHEP 03 (2014) 019 [arXiv: 1212.1586] [INSPIRE].

[27] C. Hull and B. Zwiebach, Double Field Theory, JHEP 09 (2009) 099 [arXiv:0904.4664] [INSPIRE].

[28] O. Hohm, D. Lüst and B. Zwiebach, The spacetime of double field theory: review, remarks, and outlook, Fortsch. Phys. 61 (2013) 926 [arXiv:1309.2977]. 
[29] C. Hull and B. Zwiebach, The Gauge algebra of double field theory and Courant brackets, JHEP 09 (2009) 090 [arXiv:0908.1792] [INSPIRE].

[30] O. Hohm, C. Hull and B. Zwiebach, Background independent action for double field theory, JHEP 07 (2010) 016 [arXiv: 1003.5027] [INSPIRE].

[31] O. Hohm, C. Hull and B. Zwiebach, Generalized metric formulation of double field theory, JHEP 08 (2010) 008 [arXiv: 1006.4823] [INSPIRE].

[32] C.M. Hull, Finite gauge transformations and geometry in double field theory, JHEP 04 (2015) 109 [arXiv:1406.7794] [INSPIRE].

[33] O. Hohm and H. Samtleben, Exceptional form of D=11 supergravity, Phys. Rev. Lett. 111 (2013) 231601 [arXiv: 1308.1673] [INSPIRE].

[34] O. Hohm and H. Samtleben, Exceptional field theory. II. E 7(7) , Phys. Rev. D 89 (2014) 066017 [arXiv: 1312.4542] [INSPIRE].

[35] O. Hohm and H. Samtleben, Consistent Kaluza-Klein truncations via exceptional field theory, JHEP 01 (2015) 131 [arXiv:1410.8145] [INSPIRE].

[36] D. Sullivan, Infinitesimal computations in topology, Publ. Math. IHES 47 (1977) 269.

[37] L. Castellani, R. D'Auria and P. Fré, Supergravity and superstrings: a geometric perspective, World Scientific, Singapore (1991).

[38] P.K. Townsend and P. van Nieuwenhuizen, Gauged seven-dimensional supergravity, Phys. Lett. B 125 (1983) 41 [INSPIRE].

[39] A. Salam and E. Sezgin, $\mathrm{SO}(4)$ gauging of $N=2$ supergravity in seven-dimensions, Phys. Lett. B 126 (1983) 295 [INSPIRE].

[40] E. Bergshoeff, I.G. Koh and E. Sezgin, Yang-Mills/Einstein supergravity in seven-dimensions, Phys. Rev. D 32 (1985) 1353 [InSPIRE].

[41] P. Fré, P.A. Grassi, L. Ravera and M. Trigiante, Minimal $D=7$ supergravity and the supersymmetry of Arnold-Beltrami flux branes, JHEP 06 (2016) 018 [arXiv:1511.06245] [INSPIRE]. 